\title{
Early Observations by the GOES-13 Solar Extreme Ultraviolet Sensor (EUVS)
}

\author{
J.S. Evans • D.J. Strickland • W.K. Woo • \\ D.R. McMullin · S.P. Plunkett • R.A. Viereck • \\ S.M. Hill · T.N. Woods • F.G. Eparvier
}

Received: 3 September 2009 / Accepted: 2 December 2009 / Published online: 20 January 2010

(C) The Author(s) 2010. This article is published with open access at Springerlink.com

\begin{abstract}
NOAA's GOES-13 satellite, launched in May 2006, includes a new solar sensor, called EUVS (Extreme UltraViolet Sensor), that measures energy fluxes in five broad-band spectral channels that span the region from 1 to $130 \mathrm{~nm}$. Here, we report on measurements made during the mission's six-month post-launch test (PLT) period which provided nearly continuous observations from August through November 2006 and the recording of an X9 flare that occurred on 5 December 2006. In this paper, we present a calibration model for the GOES EUVS that incorporates the effects of pointing offsets, cross-disk radiance variability (radiance refers to partial-disk emission), and changes to assumed spectral shapes. Appendices are included that report on the sensitivity to these effects. The main body of the paper gives a description of the model and data recorded during the PLT period. Comparisons are made with time-coincident measurements from TIMED/SEE (Version 10.02), SOHO/SEM, and SORCE/ SOLSTICE for the time period August-November. Comparisons are made with SORCE/XPS for the 5 December flare. In general, there is agreement among the data sets within expected measurement uncertainties. There will be a series of EUVSs extending into the next generation of GOES (starting with GOES-13). The initial performance of GOES-13 EUVS, including 5-channel measurements approximately every $11 \mathrm{~s}$ on
\end{abstract}

J.S. Evans $(\bowtie) \cdot$ D.J. Strickland · W.K. Woo

Computational Physics, Inc., 8001 Braddock Road, Suite 210, Springfield, VA 22151-2110, USA

e-mail: evans@cpi.com

D.R. McMullin

Space Systems Research Corporation, 1940 Duke Street, Suite 200, Alexandria, VA 22314, USA

S.P. Plunkett

Naval Research Laboratory, 4555 Overlook Avenue, SW, Washington, DC 20375, USA

R.A. Viereck · S.M. Hill

NOAA Space Weather Prediction Center, 325 Broadway, Boulder, CO 80305, USA

T.N. Woods · F.G. Eparvier

Laboratory for Atmospheric and Space Physics, University of Colorado, 1234 Innovation Drive,

Boulder, CO 80303-7814, USA 
a nearly continuous basis, suggests that the EUVS series will play a key role over the next many years in monitoring solar EUV variability.

\section{Introduction}

The Geostationary Operational Environmental Satellite (GOES-13) carries a multi-channel Extreme Ultraviolet Sensor (EUVS) whose solar measurements within this spectral region are new to the GOES program (Viereck et al., 2007). The observed intervals (identified below) actually fall within both the X-ray ultraviolet (XUV) region from 0.1 to $10 \mathrm{~nm}$ and the extreme ultraviolet (EUV) region from 10 to $121 \mathrm{~nm}$. GOES-13 was launched on 24 May 2006 and then underwent six months of post-launch testing (PLT). It was then placed into on-orbit storage at $105 \mathrm{~W}$ until it is needed to replace GOES-12 or GOES-11. Here, we report on the nearly continuous PLT EUVS observations over the four-month period of August-November 2006 as well as on observations of an X9 flare that occurred on 5 December 2006. There were no other X-class flares during the PLT period. The reported work, carried out over 12 months starting in October 2006, had two basic objectives. The first was to develop an EUVS calibration model that takes into account changes in calibration factors (hereafter referred to as conversion factors) arising from (1) pointing offsets across the solar disk, (2) changes in cross-disk radiance distributions (relative changes), and (3) changes in spectral shape within a given channel. The second objective was to assess the quality of the measurements. Our approach was to make comparisons with observations from the Solar EUV Experiment (SEE) on the Thermosphere Ionosphere Mesosphere Energetics and Dynamics (TIMED) satellite (Woods et al., 1998a, 2005) and with observations from the Solar EUV Monitor (SEM) on the Solar and Heliospheric Observatory (SOHO) (Judge et al., 1998, 2000a, 2000b). We have recently added additional, but smaller, sets of data from two instruments on-board the SOlar Radiation and Climate Experiment (SORCE) spacecraft, namely the XUV Photometer System (XPS) and the SOLar-STellar Irradiance Comparison Experiment (SOLSTICE). Extensive comparisons will follow with a description of the calibration model preceding them.

Viereck et al. provide a first look at EUVS data from a portion of the GOES-13 PLT phase along with basic sensor design, a description of calibration at the component level, measured response functions, and changes in response functions due to field-of-view offsets derived from the model addressed below. This paper provides a significant expansion by first documenting this model that quantifies effects arising from variations in spectral shapes, in luminosity distributions across the solar disk, and in offsets as just noted. Particular attention is given to spectral effects whose understanding is essential to the overall EUVS error budget. All useable PLT calibrated data, as obtained with the aid of the model being documented, are presented and compared with independent observations. Included are data with comparisons from an X-class flare and spectra (flare and non-flare) constructed from EUVS observations.

EUVS provides an important addition to XUV/EUV (hereafter referred to as EUV) observations from other satellite sensors flown in the past 12 years. Two of these are the abovecited SOHO/SEM and TIMED/SEE sensors, which were launched in December 1995 and December 2001, respectively. Others are the XUV sensor on-board the Student Nitric Oxide Explorer (SNOE) launched in February 1998 (Bailey et al., 2000, 2001) and a similar sensor on-board SORCE that was launched in January 2003 (Woods, Rottman, and Vest, 2005; Woods and Rottman, 2005). The SNOE, SORCE, and short wavelength SEE measurements $(<27 \mathrm{~nm})$ are made with broad-band photometers (a version of XPS on each satellite). EUVS and SEM are also broad-band sensors but use transmission gratings that record designated wavelength intervals determined by detector dimensions and locations (described 
Table 1 Column 2 shows the effective detector intervals over which total signals arise while column 3 shows intervals for reporting energy fluxes as provided early in this work by SWPC.

\begin{tabular}{lll}
\hline Channel & $\lambda_{\min } \rightarrow \lambda_{\max }(\mathrm{nm})$ & $\lambda_{\min }^{\prime} \rightarrow \lambda_{\max }^{\prime}(\mathrm{nm})$ \\
\hline $\mathrm{A}$ & $1 \rightarrow 18$ & $5 \rightarrow 15$ \\
$\mathrm{~B}$ & $5 \rightarrow 35$ & $25 \rightarrow 34$ \\
$\mathrm{C}$ & $17 \rightarrow 67$ & $42 \rightarrow 63$ \\
$\mathrm{D}$ & $17 \rightarrow 84$ & $17 \rightarrow 81$ \\
$\mathrm{E}$ & $118 \rightarrow 127$ & $118 \rightarrow 127$ \\
\hline
\end{tabular}

in detail in Appendix B). SEE measurements longward of $27 \mathrm{~nm}$ (extending to $194 \mathrm{~nm}$ ) make use of the EUV Grating Spectrograph (EGS) at a resolution of $0.4 \mathrm{~nm}$. The SEM, SNOE, SEE, and SORCE experiments were/are funded by NASA as science investigations. EUVS, on the other hand, is an operational sensor being flown on a series of GOES satellites starting with GOES-13. Through NOAA's Space Weather Prediction Center (SWPC), EUVS data will be made available to the public within seconds of the end of each exposure. This real-time capability of the sensor is critical for space weather products and services. The sensor's normal mode will provide continuous observations with an overall high duty cycle during its lifetime. These will complement measurements from the EUV Variability Experiment (EVE) to be launched in early 2010 on NASA's Solar Dynamics Observatory (Woods, Lean, and Eparvier, 2006). EVE's measurement range will be from 0.1 to $105 \mathrm{~nm}$ with a spectral resolution of $0.1 \mathrm{~nm}$ longward of $5 \mathrm{~nm}$. The new knowledge of XUV spectral behavior to be gained from EVE will lead to improved EUVS conversion factors since EUVS must assume spectral shapes in specifying these factors. On the other hand, EVE will benefit from independent measurements that are expected to be well-calibrated and to provide high-quality variability with $\sim 10$ s cadence and $100 \%$ duty cycle.

The EUVS is a set of transmission grating spectrographs built by GE Panametrics (now owned by Assurance Technology Corporation (ATC)). EUVS possesses five spectral channels designated by the letters A though E. Table 1 identifies the wavelength regions of these channels. The middle column shows the intervals defined by the detectors, while the right column shows candidate band intervals for reporting calibrated data as provided by NOAA SWPC through co-authors RAV and SMH early in this investigation. Three transmission gratings provide wavelength dispersion for five detectors recording irradiances within the channels. Detectors A and B share a grating as do detectors C and D. Detector E and the third grating form an optical unit for measuring $\mathrm{H} \mathrm{Ly} \alpha$ at $121.6 \mathrm{~nm}$. It should be emphasized, and will be further addressed in Section 3, that we are not restricted to the limits in the right column. The calibration model allows users to conveniently select their own channel definitions (reporting intervals); basically, one specifies a normalized spectrum within the region containing the signal, scales the spectrum using the measured counts, and then integrates this spectrum over the desired reporting interval. It is strongly recommended, however, that the intervals be chosen to contain a significant fraction of the total channel signal. To illustrate how total counts are distributed over regions from which signals are received, we introduce Tables 2 and 3, which provide the signal contributions in percent within $5 \mathrm{~nm}$ intervals for Channels A-D; the first $5 \mathrm{~nm}$ interval has been further subdivided into a $0-2 \mathrm{~nm}$ interval and a $2-5 \mathrm{~nm}$ interval. The signal contribution in each interval is the integral of a normalized solar spectrum multiplied by the instrument response function (see Section 3). The spectral shapes used to derive the relative contributions in Tables 2 and 3 come from a 1 July 2006 quiet-time SEE spectrum and one recorded during the 28 October 2003 X-class flare, respectively. Two figures will follow that include the spectra in energy flux units. Some of the differences between the tables might seem counterintuitive as a result of the combination of spectral shape changes over extended regions and normalizing 
Table 2 Signal contributions in percent within $5 \mathrm{~nm}$ intervals for Channels A-D with the exception of $0-5 \mathrm{~nm}$, which is subdivided to accomodate Channel A. The values total to $100 \%$ for each channel (using more significant figures than displayed). The spectral shapes used to derive the contributions come from the 1 July 2006 SEE spectrum in Figure 1. An indication of how the contributions change with spectral variations is provided in the third panels of Figures 16-19.

\begin{tabular}{ccccc}
\hline$\Delta \lambda(\mathrm{nm})$ & Channel A & Channel B & Channel C & Channel D \\
\hline $0-2$ & 1.4 & 0.0 & 0.0 & 0.0 \\
$2-5$ & 12.8 & 0.0 & 0.0 & 0.0 \\
$5-10$ & 53.8 & 0.0 & 0.0 & 0.0 \\
$10-15$ & 28.5 & 0.1 & 0.0 & 0.0 \\
$15-20$ & 3.6 & 0.9 & 13.2 & 26.7 \\
$20-25$ & 0.0 & 4.2 & 21.3 & 16.6 \\
$25-30$ & 0.0 & 50.7 & 25.5 & 15.9 \\
$30-35$ & 0.0 & 44.0 & 23.3 & 15.2 \\
$35-40$ & 0.0 & 0.0 & 0.6 & 16.9 \\
$40-45$ & 0.0 & 0.0 & 4.0 & 4.8 \\
$45-50$ & 0.0 & 0.0 & 4.4 & 2.3 \\
$50-55$ & 0.0 & 0.0 & 2.4 & 0.0 \\
$55-60$ & 0.0 & 0.0 & 3.2 & 0.0 \\
$60-65$ & 0.0 & 0.0 & 2.1 & 0.0 \\
$65-70$ & 0.0 & 0.0 & 0.0 & 0.1 \\
$70-75$ & 0.0 & 0.0 & 0.0 & 0.6 \\
$75-80$ & 0.0 & 0.0 & 0.0 & 0.6 \\
\hline
\end{tabular}

the results to yield percentages. As demonstrated by Tables 2 and 3, each interval in the right column of Table 1 contains the bulk of the signal for that channel with the exception of Channel $\mathrm{C}$, which must be extended to shorter wavelengths to meet this condition. While an improved interval for Channel $\mathrm{C}$ has not been incorporated in the results to follow, quantitative details addressing the problem appear in Appendix $\mathrm{C}$ along with a scaling factor in Section 4 that accounts for the full signal. More discussion regarding the EUVS reporting intervals is appropriate for Channels $\mathrm{A}-\mathrm{D}$ and will be provided after the introduction of the first five equations in Section 3.

In the absence of high-latitude forcing, the main heating and ionization sources to the thermosphere-ionosphere system come from the irradiance spanning the limits shown in Table 1. This region also accounts for most of the dayglow (Meier, 1991). During intense flares, the irradiance shortward of $5 \mathrm{~nm}$ can be significant and will be deposited primarily in the lower thermosphere and upper mesosphere. See, for example, Figures A2 and A3 in Strickland et al. (2007), which illustrate the wavelength-dependent altitude distribution of solar energy deposition for examples of flare and non-flare spectra. Shown are volume emission rates for the optical features OI 135.6 and $\mathrm{N}_{2} \mathrm{LBH}$ that serve to illustrate the behavior. Important insights into the global response to solar EUV energy deposition and its subsequent circulation (upwelling in heated regions and downwelling in cooler regions) have come from thermospheric general circulation modeling (see, e.g., Mayr, Harris, and Spencer, 1978; Dickinson, Ridley, and Roble, 1984; Fuller-Rowell and Rees, 1983; Burns, Killeen, and Roble, 1989). Current knowledge of solar EUV variability and its influence on Earth's upper atmosphere should be extended with the availability of high duty-cycle EUVS data over extended time periods. If spectra are desired in addressing the EUV response, they can be constructed from the channel measurements with the aid of spectral shapes, either measured or modeled (see Section 4.4).

The rest of the paper proceeds as follows. Section 2 provides more information on our data and model sources. A description of our algorithm for deriving conversion factors and energy fluxes follows in Section 3. Comparisons of August-November 2006 EUVS data 
Table 3 Same as Table 2 except that the SEE spectral shapes used to derive the contributions come from observations of the 28 October 2003 X-class flare (from orbit 10219 ).

\begin{tabular}{|c|c|c|c|c|}
\hline$\Delta \lambda(\mathrm{nm})$ & Channel A & Channel B & Channel C & Channel D \\
\hline $0-2$ & 12.7 & 0.0 & 0.0 & 0.0 \\
\hline $2-5$ & 10.1 & 0.0 & 0.0 & 0.0 \\
\hline $5-10$ & 30.7 & 0.2 & 0.0 & 0.0 \\
\hline $10-15$ & 46.0 & 0.8 & 0.2 & 0.0 \\
\hline $15-20$ & 0.5 & 0.7 & 10.9 & 21.2 \\
\hline $20-25$ & 0.0 & 3.5 & 19.6 & 16.0 \\
\hline $25-30$ & 0.0 & 51.7 & 28.3 & 18.4 \\
\hline $30-35$ & 0.0 & 43.1 & 24.1 & 16.6 \\
\hline $35-40$ & 0.0 & 0.0 & 0.6 & 19.1 \\
\hline $40-45$ & 0.0 & 0.0 & 4.3 & 5.3 \\
\hline $45-50$ & 0.0 & 0.0 & 4.5 & 2.1 \\
\hline $50-55$ & 0.0 & 0.0 & 2.7 & 0.0 \\
\hline $55-60$ & 0.0 & 0.0 & 3.0 & 0.0 \\
\hline $60-65$ & 0.0 & 0.0 & 1.7 & 0.0 \\
\hline $65-70$ & 0.0 & 0.0 & 0.0 & 0.1 \\
\hline $70-75$ & 0.0 & 0.0 & 0.0 & 0.6 \\
\hline $75-80$ & 0.0 & 0.0 & 0.0 & 0.5 \\
\hline
\end{tabular}

with SEE and SEM data are given in Section 4 along with EUVS energy fluxes for the 5 December 2006 X-class flare. A summary completes the main body of the paper in Section 5. There are three appendices. The first contains a list of terms and units as supplemental information to Section 3. Appendices B and C address the dependence of conversion factors on pointing offsets, cross-disk radiance distributions, and spectral shapes.

\section{Data Sources and Solar Irradiance Models}

The sensors providing solar data addressed in this study are the following with EUVS, SEE, SEM, and the SORCE sensors having been already introduced in the previous section:

- GOES-13/EUVS

- GOES-13/SXI

- GOES-12/XRS

- $\mathrm{TIMED} / \mathrm{SEE}$

- SOHO/SEM

- SORCE/XPS

- SORCE/SOLSTICE

All available PLT uncalibrated EUVS data were provided by SWPC. The data rate for each channel is approximately one measurement per $11 \mathrm{~s}$. SXI (Solar X-ray Imager) images, also provided by SWPC, are of interest for EUVS pointing knowledge (SXI and EUVS are coaligned on the yoke assembly to which solar array panels are mounted) and for specifying normalized cross-disk radiance distributions to be used as proxies for the EUVS spectral regions. The use of such distributions is taken up in Appendix B to investigate the sensitivity of conversion factors to deviations from uniform radiance. GOES-12 XRS (X-Ray Sensor) full-disk data are of interest for comparing their temporal behavior during the 5 December 
2006 X9 flare with those from the five EUVS channels. The XRS data ( 0.1 to $0.8 \mathrm{~nm}$ ) were downloaded from NOAA's National Geophysical Data Center.

SEE data play dual roles, firstly, to provide spectral characterization for converting EUVS counts to energy flux and secondly, for making comparisons with EUVS energy fluxes over extended time periods. SEE Version 10.02 data in units of $\mathrm{W} \mathrm{m}^{-2} \mathrm{~nm}^{-1}$ were downloaded from the SEE ftp site (ftp://laspftp.colorado.edu/pub/SEE_Data/level3a/). The wavelength resolution is $1 \mathrm{~nm}$ starting at $1 \mathrm{~nm}$ and extending past $\mathrm{H}$ Ly $\alpha$ at $121.6 \mathrm{~nm}$. SEE's duty cycle is one 3-minute spectral measurement per TIMED orbital period (97 min). Expanding on comments from the previous section, measurements longward of $27 \mathrm{~nm}$ are obtained by EGS, an ultraviolet grating spectrograph with a microchannel plate array detector. Measurements shortward of $27 \mathrm{~nm}$ are obtained by XPS that makes broad-band photodiode observations. These observations are converted to spectra by assuming spectral shapes within the regions covered by the photodiodes (Woods et al., 2005, 2008).

SEM data in units of photons $\mathrm{cm}^{-2} \mathrm{~s}^{-1}$ were downloaded from the SEM website (http://www.usc.edu/dept/space_science/semdatafolder/semdownload.htm) and then converted to $\mathrm{W} \mathrm{m}^{-2}$. The SEM spectral region is similar to that of Channel $\mathrm{B}$ and is dominated by emission from He II $30.4 \mathrm{~nm}$. Like EUVS, its signal comes from light dispersed by a transmission grating with the detector designed and positioned to record photons over the desired interval. Also like EUVS, a spectral shape must be assumed in order to convert its signal to energy flux. To do this, the SEM algorithm uses the readily available SOLERS22 spectral distribution (Woods et al., 1998b) to first convert the measured observations into photon flux. The same spectral shape is then used to convert the photon flux into energy flux $\left(\mathrm{W} \mathrm{m}^{-2}\right)$. SEM data are sampled by the SOHO/CELIAS Digital Processing Unit (DPU) at a rate of $15 \mathrm{~s}$ for all channels. The SOHO spacecraft is observing from an orbit around the Lagrange point L1 and, thus, does not orbit around the Earth. The solar observations are made continuously, without daily or even seasonal eclipses. For the present work, 5-minute averaged measurements have been compiled. For these 5 minute data sets, the day begins at 0:00:00 UT and is reported at the mid-point of 5 minutes into the day, and is the average of all data collected during the 5 minute window.

XPS on-board SORCE makes measurements similar to SEE/XPS (Woods, Rottman, and Vest, 2005; Woods and Rottman, 2005). SORCE/XPS data are used to construct spectra from 1 to $40 \mathrm{~nm}$ that are then integrated over EUVS's reporting intervals for Channels A and B. The spectra of interest to this investigation come from flare observations on 5 December 2006.

SOLSTICE, also on-board SORCE (McClintock, Rottman, and Woods, 2005; McClintock, Snow, and Woods, 2005), is providing Ly a measurements in place of SEE's for comparisons with EUVS Channel E measurements. The SOLSTICE values, while at a somewhat lower duty cycle, are preferred because of a higher signal-to-noise ratio.

Above, it was noted that one of SEE's roles is to provide spectral characterizations within EUVS channels for deriving conversion factors. Other characterizations used in the work being reported come from the following models:

- NRLEUV (Warren, Mariska, and Lean, 2001; Lean et al., 2003)

- SOLERS22 (Woods et al., 1998b).

- FISM (Flare Irradiance Spectral Model) (Chamberlin, Woods, and Eparvier, 2007).

From the NRLEUV model come two spectra that address preflare and flare conditions for the Bastille Day flare (Meier et al., 2002). The SOLERS22 spectrum is intended to represent mid-levels of solar activity. The FISM model has provided time-dependent spectra during the 5 December flare from which conversion factors and in turn EUVS energy fluxes have 

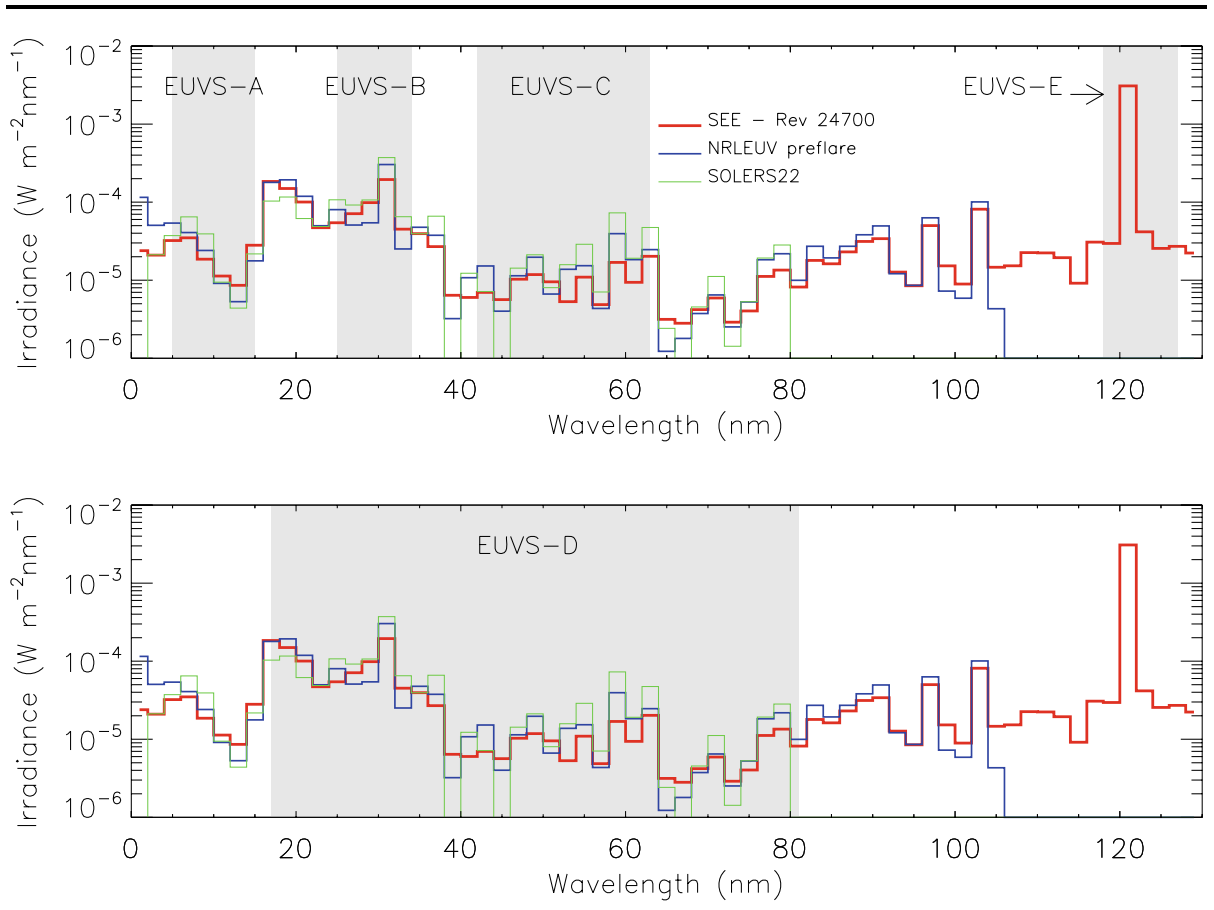

Figure 1 Top panel: Non-flare Version 10.02 SEE spectrum from 1 July 2006 (TIMED orbit 24 700) and non-flare spectra from the NRLEUV and SOLERS22 models displayed at a resolution of $2 \mathrm{~nm}$. Shown in the shaded regions are candidate reporting intervals from Table 1 for Channels A, B, C, and E. Bottom panel: The same spectra with the interval from Table 1 for Channel D.

been specified (see Section 4.3). FISM is an empirical model spanning the region from 0.1 to $193 \mathrm{~nm}$ at $1 \mathrm{~nm}$ wavelength resolution and 1 spectrum/min temporal resolution.

The NRLEUV preflare and SOLERS22 spectra may be seen in Figure 1 along with that from a quiet-time SEE spectrum from 1 July 2006 (all displayed at $2 \mathrm{~nm}$ resolution). SEE spectra are referenced to the TIMED orbit of the observations and for this example, it is orbit 24700 . Two panels are shown, each with the same spectral information. The purpose of the two panels is to clearly identify the EUVS channels as given in the right column of Table 1. The bulk of the EUVS energy fluxes presented in this paper are based on spectral characterizations from SEE. The primary role of NRLEUV and SOLERS22 is to specify EUVS energy fluxes in the sensitivity study reported in Appendix C. FISM, along with SEE and NRLEUV spectral characterizations, provide EUVS energy fluxes just before and during the 5 December flare.

\section{Algorithm for Deriving EUVS Conversion Factors}

This section describes the steps leading to derived EUVS conversion factors and how they are used to convert EUVS count rates to energy fluxes. We divide model parameters (single or multi-valued) leading to the specification of conversion factors into static and dynamic categories. Examples in the static category are filter functions, detector functions, and those sensor parameters that are geometry related (e.g., detector locations). Dynamic parameters include the following: 
Table 4 Values of $B_{n}, G_{n}$, and $V_{n}$ for deriving $Q_{n}$ from Equation (1).

\begin{tabular}{lllll}
\hline Channel & Index $n$ & $\begin{array}{l}B_{n} \\
\text { Counts }\end{array}$ & $\begin{array}{l}G_{n} \\
\text { Amps/Count }\end{array}$ & $\begin{array}{l}V_{n} \\
\text { Amps }\end{array}$ \\
\hline A & 1 & 25060 & $1.91 \times 10^{-15}$ & $2.13 \times 10^{-14}$ \\
B & 2 & 16030 & $1.89 \times 10^{-15}$ & $1.21 \times 10^{-14}$ \\
C & 3 & 16229 & $1.90 \times 10^{-15}$ & $4.79 \times 10^{-14}$ \\
D & 4 & 24387 & $1.89 \times 10^{-15}$ & $1.20 \times 10^{-15}$ \\
E & 5 & 25211 & $1.90 \times 10^{-15}$ & $1.32 \times 10^{-12}$ \\
\hline
\end{tabular}

- $\alpha_{\mathrm{o}}$, the East - West offset of the solar disk within the field-of-view

- radiance distribution across the solar disk (relative variation), and

- normalized EUV spectrum over the region used for deriving a conversion factor

The first of these arises from pointing errors, while the remaining two arise from solar variability. The motivation for developing the reported model was to quantify the effects on conversion factors due to their possible changes. There is a weak effect due to changes in the radiance distribution (addressed in Appendix B.4) and thus, our primary focus will be on the remaining two terms.

The key computational step is the derivation of the geometry function $f_{\text {geom }}\left(m, \lambda, \alpha_{\mathrm{o}}\right)$, namely the fraction of the energy flux entering EUVS from the full solar disk that reaches the active area of the detector for order $\mathrm{m}$, wavelength $\lambda$, and $\alpha_{\mathrm{o}}$. This function leads to channelspecific wavelength restrictions. Appendix B.2 describes how the function is calculated.

\subsection{Introduction of Conversion Factors and Their Use in Deriving EUVS Energy Fluxes}

Conversion factors are the end-products from our model and are used to convert channelspecific EUVS count rates to energy fluxes. It is here that the need arises for specifying a spectral shape over the region that contributes to the signal within the channel of interest. The signal, in effect, provides an absolute magnitude to the spectrum, after which the energy flux may be conveniently specified over a wavelength interval of the user's choosing. Table 1, previously introduced in Section 1, helps to clarify the point by showing the effective spectral region per channel contributing to that channel's total signal (required for calculating conversion factors) and a candidate interval for reporting energy fluxes. There is nothing unique about these latter intervals, but it is recommended that they stay within the bounds of the intervals used to calculate conversion factors and include the regions making significant contributions to the total signals (this, in fact, is not the case for Channel $\mathrm{C}$, as will be shown in Appendix C).

The conversion factor for the $n$th channel is designated as $C_{n}$ in units of $\mathrm{A} /\left[\mathrm{W} \mathrm{m}^{-2}\right]$. It converts EUVS count rates to energy fluxes using

$$
Q_{n}=\left[\left(S_{n}-B_{n}\right) G_{n}-V_{n}\right] C_{n}^{-1} \mathrm{~W} \mathrm{~m}^{-2}
$$

with the remaining terms being energy flux, $Q_{n}$, total signal, $S_{n}$ in counts over the EUVS integration period of approximately $11 \mathrm{~s}$, background, $B_{n}$ in counts, gain, $G_{n}$ in Amps/count, and visible light contamination, $V_{n}$ in Amps. Table 2 in Viereck et al. provide values of $B_{n}, G_{n}$, and $V_{n}$, which are repeated here in Table 4 for the convenience of the reader.

$C_{n}$ is derived from 


$$
\begin{aligned}
C_{n} & =\int_{\lambda_{\min }^{n}}^{\lambda_{\max }^{n}} f_{\mathrm{AR}}^{n}(\lambda) \phi_{N}^{n}(\lambda) \mathrm{d} \lambda \\
& =\sum_{j} \int_{\lambda_{j}}^{\lambda_{j}+\delta \lambda} f_{\mathrm{AR}}^{n}(\lambda) \phi_{N}^{n}(\lambda) \mathrm{d} \lambda \\
& =\sum_{j} \phi_{N}^{n, j} \int_{\lambda_{j}}^{\lambda_{j}+\delta \lambda} f_{\mathrm{AR}}^{n}(\lambda) \mathrm{d} \lambda \\
& =\sum_{j} \phi_{N}^{n, j} f_{\mathrm{IR}}^{n, j}
\end{aligned}
$$

with terms defined as follows:

$\lambda_{\min }^{n}, \lambda_{\max }^{n} \quad$ Limits over effective range of full signal

$f_{\mathrm{AR}}^{n}(\lambda) \quad$ Absolute response function in $\mathrm{A} /\left[\mathrm{W} \mathrm{m}^{-2}\right]$

$\phi_{N}^{n}(\lambda) \quad$ Assumed EUV spectrum normalized between $\lambda_{\min }^{n}$ and $\lambda_{\max }^{n}$ in $\mathrm{nm}^{-1}$

$\delta \lambda \quad$ Resolution for converting integral to a sum

$f_{\mathrm{IR}}^{n}(\lambda) \quad$ Integrated response function in $\mathrm{A} /\left[\mathrm{W} \mathrm{m}^{-2} \mathrm{~nm}^{-1}\right]$.

It is important to note that the integral over wavelength is ideally over all wavelengths but that a limited, yet wide, range is implemented based on the calibration range for the absolute response function. The bulk of the computations within our model are for specifying $f_{\mathrm{AR}}^{n}(\lambda)$ as described in Section 3.2 and in Appendix B.2. The sums in Equation (2) are on a $1 \mathrm{~nm}$ grid in the work being reported. When a TIMED/SEE spectrum is used to derive $\phi_{N}^{n, j}$, its unnormalized values $\phi_{j}$ (in $\mathrm{W} \mathrm{m}^{-2} \mathrm{~nm}^{-1}$ ) are already on a $1 \mathrm{~nm}$ grid that requires summing only $\phi_{j}$ values between $\lambda_{\min }^{n}$ and $\lambda_{\max }^{n}$ to obtain the normalization constant $\phi_{T, n}$ for specifying the $\phi_{N}^{n, j}$ values from

$$
\phi_{N}^{n, j}=\frac{\phi_{j}}{\phi_{T, n}} .
$$

Otherwise, the applied spectrum $\phi(\lambda)$ must be appropriately converted in both its units and in resolution followed by its normalization.

Once $Q_{n}$ is derived from Equation (1) for a given measurement, an absolute spectrum can be derived as follows:

$$
\phi_{\mathrm{EUVS}}^{n, j}=Q_{n} \phi_{N}^{n, j}
$$

It is worth noting that the same $\phi_{\mathrm{EUVS}}^{n, j}$ values are obtained for different sets of $\lambda_{\min }^{n}$ and $\lambda_{\max }^{n}$ as long as each of the intervals contains all of the non-zero values of $f_{\mathrm{IR}}^{n, j}$. A wider interval decreases $\phi_{N}^{n}$ and is compensated by a larger value of $Q_{n} . \phi_{\mathrm{EUVS}}^{n, j}$ provides complete flexibility for redefining limits on the $n$th channel. This is done with Equation (5) for the desired interval $\Delta \lambda^{n}$, which is recommended to be within the original $\lambda_{\min }^{n}$ to $\lambda_{\max }^{n}$ interval.

$$
Q_{n}\left(\Delta \lambda^{n}\right)=\sum_{\Delta \lambda^{n}} \phi_{\mathrm{EUVS}}^{n, j}
$$

Channel B provides an example of channel redefinition where non-zero response functions begin at $5 \mathrm{~nm}$ and extend to $35 \mathrm{~nm}$ (limits come from Table 1). The dominant signal comes from the region between 23 and $35 \mathrm{~nm}$ (based on the integrand of its conversion factor, 
examples of which are presented in Appendix C; also see Tables 2 and 3). Equation (5) can be used to conveniently restrict the reported energy flux to this or some similar interval.

We have already noted that there is no unique set of reporting intervals, but that guidance for their choice should come from the wavelength distribution of counts. We see in Table 1 that the reporting intervals for Channels $\mathrm{A}, \mathrm{B}$, and $\mathrm{C}$ are narrower than those for deriving conversion factors (i.e., those containing the full set of counts). Channel B was just singled out as an example where, nevertheless, most of the counts come from its reporting interval in the table. This is also true of Channel A. Channel C, on the other hand, captures a small fraction of the counts in its reporting interval as already noted in Section 1 and discussed in detail in Appendix C. Its interval should be extended down to $\sim 15 \mathrm{~nm}$ based on panel 3 in Figure 18. Channel $\mathrm{C}$ energy fluxes to follow make use of the reporting interval in the table but a scaling factor is provided to convert them to the interval containing the full signal. One might argue for also extending the reporting intervals for Channels A and B to shorter wavelengths, particularly for application to flare data. There is no obstacle to doing this with Equation (5), but it should be kept in mind that there may be a significant contribution to the energy flux from, say, 1 to $5 \mathrm{~nm}$ for Channel A (for intense flares) and from 5 to $25 \mathrm{~nm}$ for Channel B (all conditions) in spite of little relative EUVS signal from these regions. The absolute magnitudes of the discrete spectra being summed are dictated by the signals at longer wavelengths (under both flare and non-flare conditions). The success of reporting accurate enhancements of energy fluxes by such extensions depends on how physically realistic the assumed spectral shape is in the extended region compared to its shape and magnitude in the region containing the bulk of the signal. When in doubt, caution is advised in reporting from extended regions contributing little to the total signal but significantly to the energy flux.

A reverse of the above situation for Channel $\mathrm{C}$ occurs for Channel $\mathrm{D}$ whose reporting interval in Table 1 extends to longer wavelengths, where there is little signal. Tables 2 and 3 show that $\sim 99 \%$ of the signal occurs between 15 and $50 \mathrm{~nm}$. Thus, the utility of the portion of the reported energy flux from 50 to $81 \mathrm{~nm}$ depends on the reliability of the assumed spectral shape beyond $50 \mathrm{~nm}$ relative to that at shorter wavelengths.

\subsection{Calculating Absolute Response Functions}

An absolute response function is determined from the wavelength-dependent properties of the transmission grating, the detector, a filter, and the geometry function. A filter function is provided for a stand-alone filter which, in the case of EUVS, only applies to Channel E. For Channels A-D, the filters are coatings on the detectors with their attenuation properties incorporated into detector functions. In these cases, the stand-alone filter function within model calculations is set to unity. The derivation of the absolute response function for the $n$th channel is provided by

$$
f_{\mathrm{AR}}^{n}\left(\lambda, \alpha_{\mathrm{o}}\right)=f_{\text {det }}^{n}(\lambda) f_{\text {filter }}^{n}(\lambda) f_{\text {grating }}^{n, m}(\lambda) f_{\text {geom }}^{n, m}\left(\lambda, \alpha_{\mathrm{o}}\right) \mathrm{A} /\left[\mathrm{W} \mathrm{m}^{-2}\right]
$$

where $f_{\text {det }}^{n}$ is the detector function (i.e. sensitivity) in $\mathrm{A} /\left[\mathrm{W} \mathrm{m}^{-2}\right], f_{\text {filter }}^{n}$ is the stand-alone filter function (dimensionless), $f_{\text {grating }}^{n, m}$ is the transmission grating function (dimensionless) that is order dependent, thus introducing the second superscript $m$, and $f_{\text {geom }}^{n, m}$ is the geometry function (also dimensionless). The short and long wavelength cutoffs of $f_{\mathrm{AR}}$ are determined primarily by $f_{\text {geom }}$ as can be seen from the examples given in Figure 15 . Note that a similar equation appears in Viereck et al., which shows an unintended sum rather than a product on the right hand side. 
For Channels $\mathrm{A}-\mathrm{D}, f_{\text {det }}^{n}$ is based on a simple layering model and the transmission properties of the combined filter and detector components (Henke, Gullikson, and Davis, 1993). For Channel E, the same approach is used except that a separate characterization is given to the stand-alone filter function. The transmission grating function $f_{\text {grating }}$ (order dependent) is based on initial-order calculations for a typical 5000 line $/ \mathrm{mm}$ grating. The resultant values were compared with measurements of similar gratings and found to be reasonable (Hettrick et al., 2004).

Adjustments have been made to the calculated grating functions in order to achieve approximate agreement with measured values of $f_{\mathrm{AR}}$ (introduced shortly). One limitation of using a calculation for the grating transmission is that the results will produce a theoretically perfect response for the given parameters that do not include grating imperfections. While the expected response for calculated first-order transmissions was very close to that measured in similar gratings, the calculated even-order transmission is significantly smaller than measured even-order responses observed in similar gratings (McMullin et al., 2004; Ceglio et al., 1988). This has been attributed to the slight imperfections in the 1:1 gap-tobar ratio of the flight gratings. In theory, the calculated transmission for even orders of a 1:1 grating is zero. However, since measurements have shown that this is not the case, the EUVS model grating component $f_{\text {grating }}$, was modified for even-order transmission to achieve values similar to those reported by Hettrick et al. (2004) for gratings with the same properties. When combined with the remaining channel parameters, the end-to-end modeled channel efficiencies agree very well with the measured values (presented shortly). Our overall approach was to first achieve this agreement and then extend the model to non-zero offsets. It is important to note, however, that the same order-dependent grating functions are used for Channels A and B since they possess a common grating. The same constraint applies to Channels $\mathrm{C}$ and $\mathrm{D}$.

Calculated response functions for Channels $\mathrm{A}-\mathrm{E}$ and for all orders considered (see Table 9) may be seen in Figure 2 along with measured values, again for $\alpha_{\mathrm{o}}=0^{\circ}$ (the latter values also appear in Viereck et al. within a single frame). There are four different wavelength scales reflecting the different spectral regions spanning the five channels. The Channel B and $\mathrm{D}$ geometry functions for first and second order do not allow for non-zero values of $f_{\mathrm{AR}}$ from $\sim 17$ to $\sim 24 \mathrm{~nm}$ and from $\sim 47$ to $\sim 68 \mathrm{~nm}$, respectively. The measured values in these regions are consistent with the possibility of wavelength impurities or scattered light effects during calibration. At the shortest wavelengths for Channel B, measured values extend farther than our calculated values. Unlike Channel A, where the instrument response has been modeled to sixth order to account for possible higher-order contributions below $5 \mathrm{~nm}$ (especially during flares), modeling of the Channel B instrument response beyond third order is deemed unnecessary. The Channel B measured response shortward of $8 \mathrm{~nm}$ is almost two orders of magnitude below the first-order response, where, as Tables 2 and 3 show, nearly all the signal resides due to He II $30.4 \mathrm{~nm}$. The non-zero values in the measured response longward of $80 \mathrm{~nm}$ in Channel D are consistent with wavelength impurities that allow photons at shorter wavelengths into the instrument, specifically photons longward of $40 \mathrm{~nm}$ that reach the detector in second order.

\section{Early Mission EUVS Energy Flux Measurements}

The bulk of PLT EUVS data are presented below, most of them during the four-month period from August through November 2006 when data were recorded on essentially a continuous basis. Measurements were very intermittent during the other two PLT months (July and December) due to scheduled test maneuvers. Nevertheless, EUVS was recording data during 

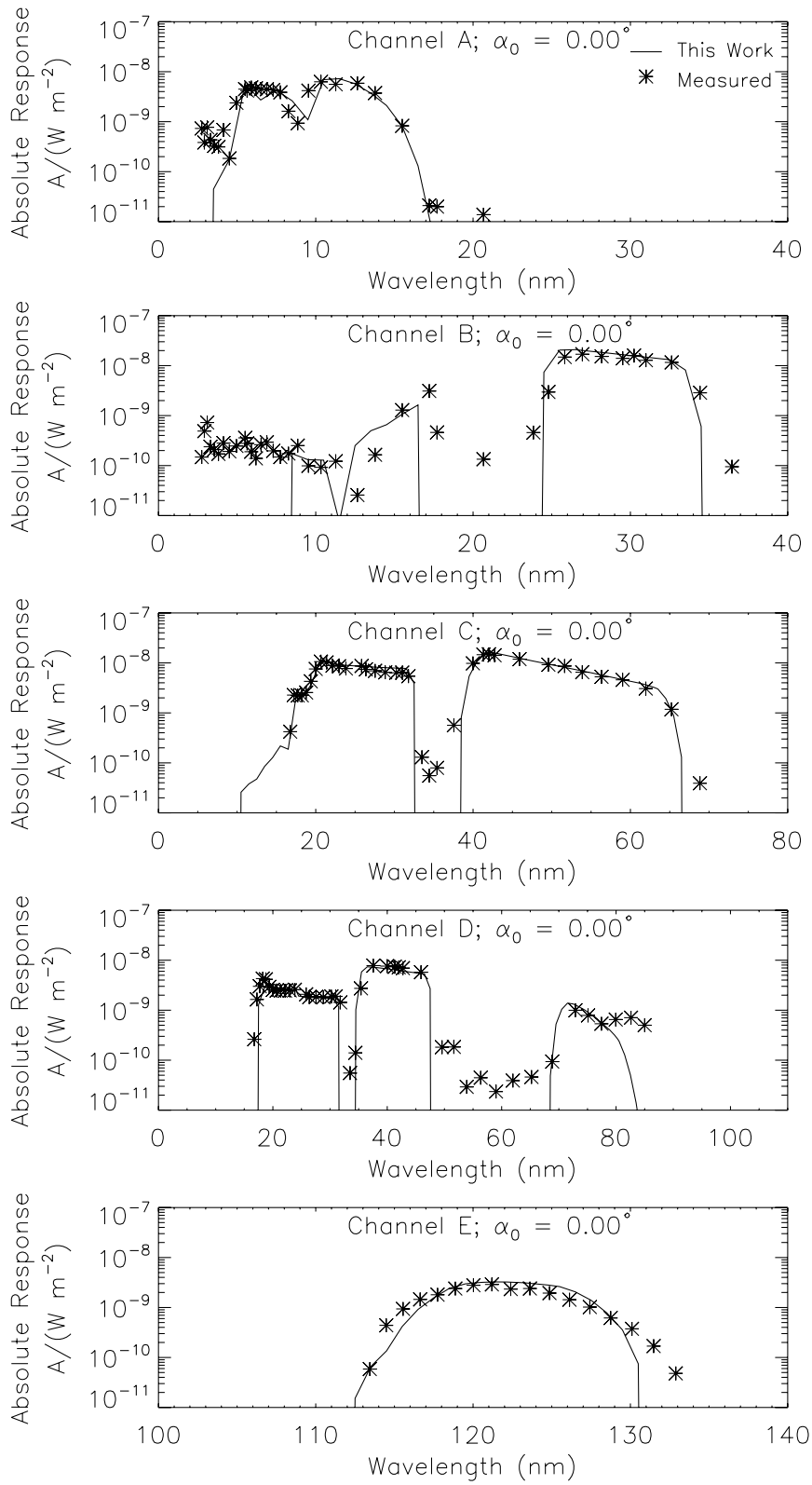

Figure 2 Derived and measured absolute response functions for Channels $\mathrm{A}-\mathrm{E}$ at $1 \mathrm{~nm}$ resolution and $\alpha_{\mathrm{O}}=0^{\circ}$. Adjustments have been made to grating functions to improve agreement with the measurements.

the 5 December 2006 flare that will also be addressed. We begin with error budget considerations (Section 4.1), then present the August-November data along with comparisons to SEE, SEM, and SOLSTICE measurements (Section 4.2), then the X9 flare data (Section 4.3), and finally show a first step in constructing spectra from the multi-channel EUVS data. 


\subsection{Error Budget Considerations}

Appendices B and C are important to conclusions about EUVS data quality based on the comparisons in Section 4.2. These appendices illustrate the sensitivity of conversion factors $C_{n}$ and energy fluxes $Q_{n}\left(\Delta \lambda^{n}\right)$ to $\alpha_{\mathrm{o}}$, cross-disk radiance distributions, and spectral shapes, respectively. They do not, however, extend the studies to the point of specifying uncertainties in $Q_{n}\left(\Delta \lambda^{n}\right)$, which is a significant challenge with regard to the current state of knowledge of spectral behavior. We can say, however, based on Appendix B.3, that variations in radiance distributions (for absolute offset values less than $0.5 \mathrm{deg}$ ) are unimportant relative to the overall error budget for conversion factors.

The EUVS fluxes presented in Section 4.2 are based on an assumed offset and on the spectral behavior taken from the orbit 24700 quiet-time SEE spectrum in Figure 1. There will be time-dependent channel biases (biases referenced to true values, not to other measured values) reflected in any unaccounted-for temporal variations in $\alpha_{\mathrm{o}}$ and spectral shapes. Knowledge is available on temporal variations in $\alpha_{\mathrm{o}}$ (monitored with the aid of SXI images) and, based on an initial assessment that takes results from Appendix B.3 into account, pointing errors should make a minor contribution to the overall error budget.

The results in Appendix $\mathrm{C}$ suggest that uncertainties in spectral shapes make a significant contribution to the error budget. Temporal biases relative to their averages, however, are probably small over the quiet period from August through November. Some of the complications arising from spectral uncertainties are discussed in Appendix C. Large errors can occur where spectral shapes change in regions of relatively high but structured responsivity. Channels $\mathrm{B}$ and $\mathrm{E}$ are the exceptions due to the dominance of single features that minimize the effect of surrounding spectral changes within these channels. Channels A, C, and D all contain important contributions to their signals shortward of $\sim 30 \mathrm{~nm}$. Using SEE spectral shapes in this region rely on XPS measurements that, themselves, require assumptions about the spectral behavior. Nevertheless, the spread in Channel A energy fluxes reported in Appendix $\mathrm{C}$ among SEE and other representations (flare and non-flare) are modest $(\sim 25 \%)$. While this does not rule out larger uncertainties, it may provide a reasonable guide under most conditions. A smaller spread is seen for Channel D that can be attributed to signal from a broader region over which differences from one spectral representation to the next get averaged out to some extent. Channel $\mathrm{C}$ represents a special case if one selects a reporting interval similar to that in Table 1. This is discussed in some detail in Appendix C, where it is noted that most of the signal is recorded shortward of this interval. As a consequence, the magnitude of the spectral segment within this interval is not determined by the signal there and thus leads to a larger spread than for the other channels. We recommend a redefinition of its reporting interval by one that contains significant signal.

Instrumental uncertainties are primarily tied to the measured response functions shown in Figure 2. As discussed in Section 3.2, adjustments were made, where necessary, to transmission grating curves so that the calibration model achieves the agreement shown in that figure for no pointing offset. Systematic uncertainties are difficult to quantify and have not been attempted for either response functions or spectral effects, although Appendix C does provide useful insights with regard to the latter.

\subsection{Comparisons with TIMED/SEE and SOHO/SEM}

\subsubsection{Introducing Figures 3-6}

As noted in Section 4.1, we have processed all EUVS data from August through November 2006 based on a single spectral shape per channel taken from the SEE spectrum for orbit 
24 700. The applied offset $\alpha_{\mathrm{o}}$ is $+0.23^{\circ}$ for all channels based on a NOAA-provided analysis of the SXI to EUVS co-alignment (sponsored NOAA study by Swales Aerospace). Uniform radiance across the solar disk is assumed but, based on results from Appendix B.4, significant deviations from uniformity lead to changes of less than $15 \%$ in conversion factors for the applied offset. For channels A-D, SEE spectra, in $\mathrm{W} \mathrm{m}^{-2} \mathrm{~nm}^{-1}$ at a duty cycle of one spectrum per $97 \mathrm{~min}$, have been integrated over the same intervals used for reporting EUVS energy fluxes (see column 3 in Table 1). As discussed in Section 2, broad-band photometer data (from XPS) are used to construct that portion of SEE spectra shortward of $\sim 27 \mathrm{~nm}$, while longward of this wavelength, characterization comes from spectral measurements by EGS with greater assigned accuracy $(\sim 15 \%)$. As also discussed in Section 2, SEM data are being reported as 5 minute averages and will be compared to Channel B EUVS energy fluxes while for Channel E, SORCE/SOLSTICE data provide the comparisons due to a better signal compared to SEE.

The comparisons among these data sets for the four months may be seen in Figures 3-6 (Viereck et al. provide comparisons for August with EUVS energy fluxes derived from an earlier version of our calibration model and using SEE rather than SOLSTICE data for Channel E). In each figure, Channel A comparisons appear in the top panel with comparisons for Channels $\mathrm{B}-\mathrm{E}$ in the next four followed by the solar proxy $\mathrm{F}_{10.7}$ in the bottom panel. Comparisons with both SEE and SEM are seen in the second panel of each figure for Channel B. It is worth noting that there are different relative ranges in energy flux for the five channels. Thus, the same vertical separation between EUVS and SEE data from one panel to the next does not give the same relative difference. Occasional gaps over the four months are seen for all three sensors. Minor flare activity can be seen in EUVS and SEM data during the second half of August. Minor daily dips in Channel E data (present in all months) arise from Earth occultations that, as expected, are most prominent near fall equinox when the solar declination is near $0^{\circ}$. Occultations are also responsible for the vertical distributions of data points for all channels that are aligned with the dips (unlike Channel E, not present at all times). Note that Channel E occultations are not only due to the solid earth but to the extended geocorona as well. Other narrow vertical distributions arise from off-pointing test maneuvers and periodic calibrations of the electronics.

In general, the reported EUVS fluxes are within the combined uncertainties of EUVS measurements and those of the other instruments. This finding is in significant contrast to those reported by Guha, Jones, and Benner (2007). The EUVS-determined fluxes for all channels were reported in Guha, Jones, and Benner (2007) as "orders of magnitude lower due to the attenuation by the grating and filters associated with each channel." It is not likely that attenuation by the grating and filters would have changed from the EUVS pre-flight measurements enough to account for orders of magnitude lower reported fluxes. The results presented in this paper are consistent with the pre-flight measurements and the reported fluxes measured during flight are producing results consistent with independent measurements from other instruments.

At this time it is unclear as to the source of the discrepancy, because the Guha paper discusses the model agreement with the measured response functions. If there is agreement, then the difference must be in the irradiance conversion algorithm that also uses a TIMED/SEE spectrum for specifying spectral behavior within the various channels.

\subsubsection{Max/Min EUVS Energy Flux Ratios over the August-November Solar Rotations}

Solar EUV variability on time scales from minutes to years is of considerable interest to understanding thermospheric and ionospheric variability. Short-term variability due to weak 


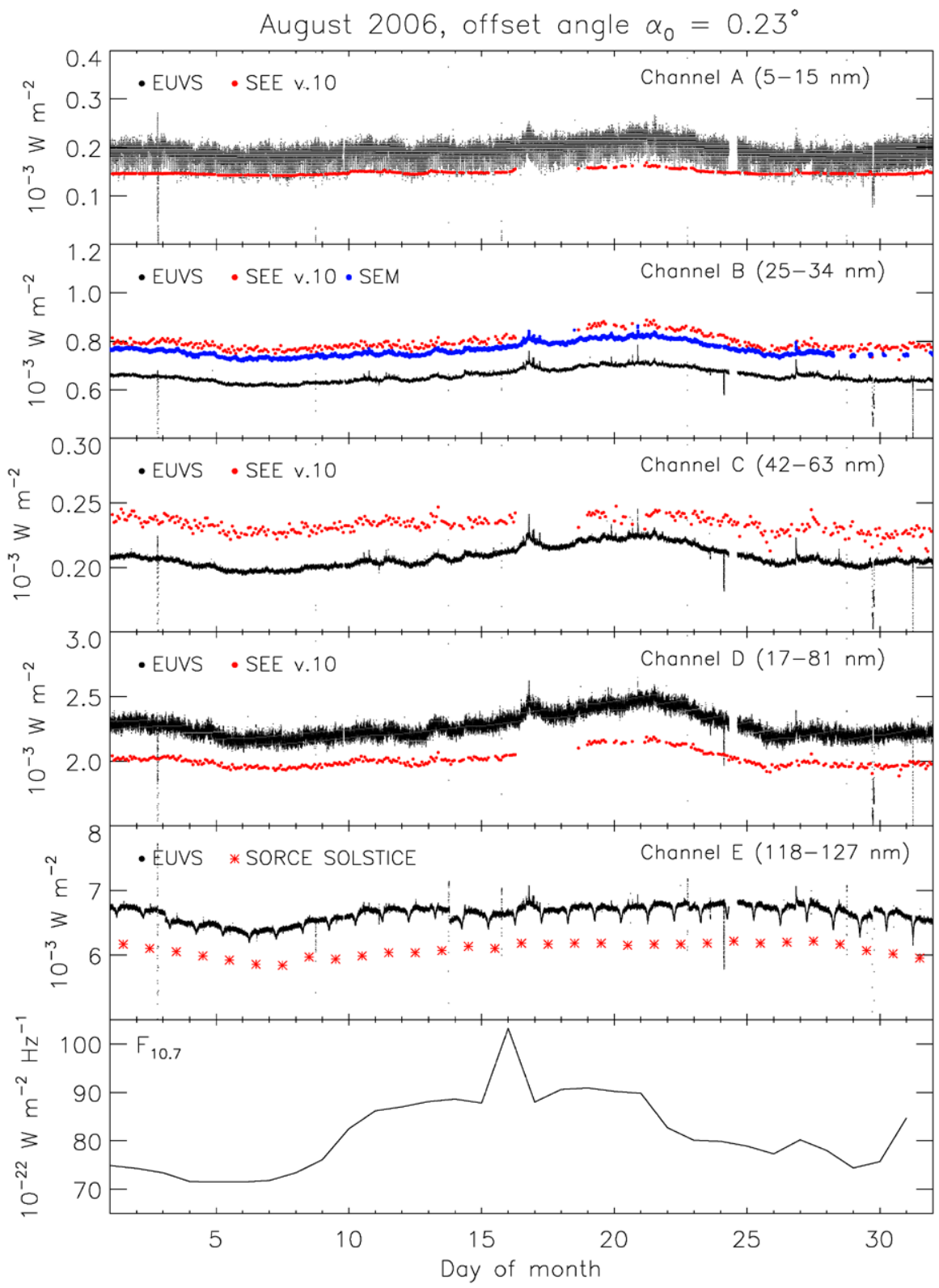

Figure 3 EUVS, Version 10.02 SEE, SEM, and SOLSTICE data over the identified wavelength intervals for August 2006. Only for Channel B may EUVS data be compared with those from both SEE and SEM. The bottom panel shows the daily variation of the $\mathrm{F}_{10.7}$ solar proxy.

flares is clearly seen in the EUVS data during August. The focus here, however, is on the variability seen in Figures 3, 4, 5 and 6 over each of the four solar rotations that occurred between August and November. Table 5 gives the EUVS max/min energy flux ratio for each channel by rotation with the time intervals of the four rotations given below the table. The 


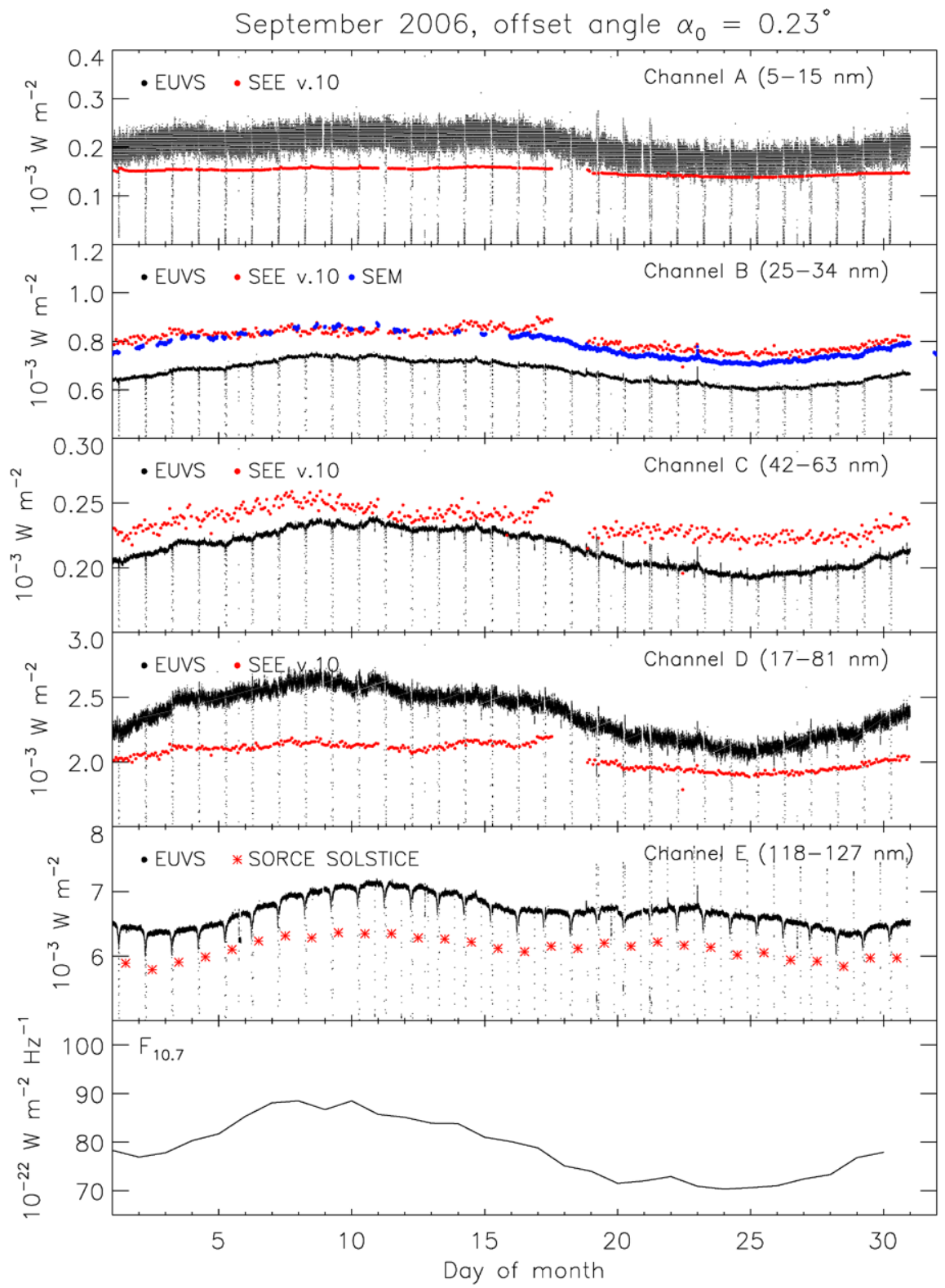

Figure 4 Comparisons for September 2006.

true flux ratios may depart from Table 5 since, per channel, a single spectral shape was applied to the conversion from counts to energy flux units. Any departures are not expected to be significant, however, under the quiet conditions that existed during the given four months. The ratios in Table 5, while identified as energy flux ratios, are also count ratios due to the use of a single spectral shape per channel. Greater variability is expected with decreasing wavelength, which can be seen by comparing ratios between channels $\mathrm{A}$ and $\mathrm{E}$. 


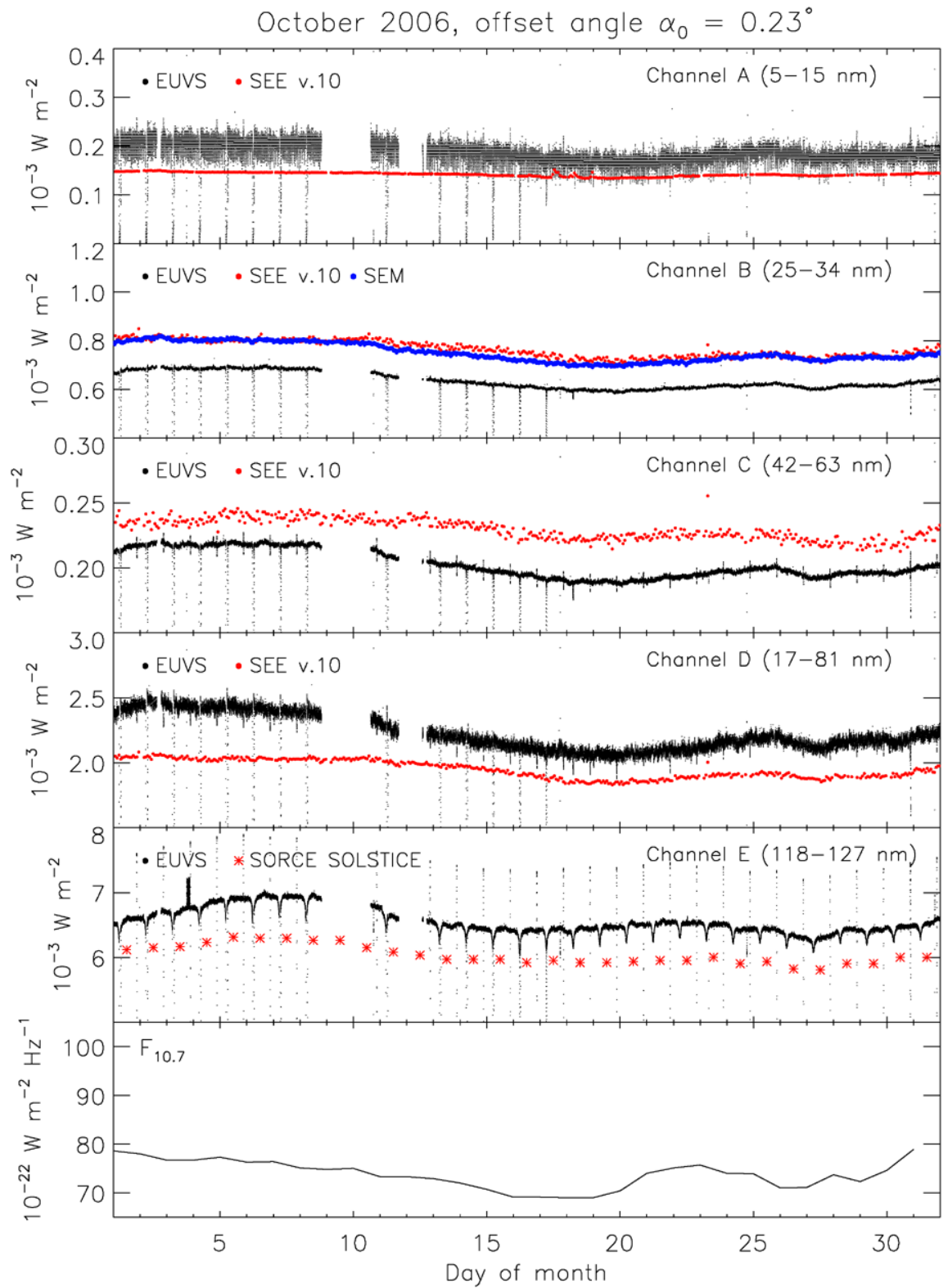

Figure 5 Comparisons for October 2006.

There is a change of $25 \%$ in Channel A during Rotation 2, to be compared to a maximum change in Channel E of only 7\% (Rotations 2 through 4). The variabilities for Channels $\mathrm{B}-\mathrm{D}$ are similar and follow from the bulk signal for each of these channels coming from a similar spectral region (in the vicinity of 20 to $35 \mathrm{~nm}$; see the third panels in Figures 17 - 19). Similar variability in the SEE, SEM, and SORCE measurements is seen with the exception of Channel A, which is addressed next. 


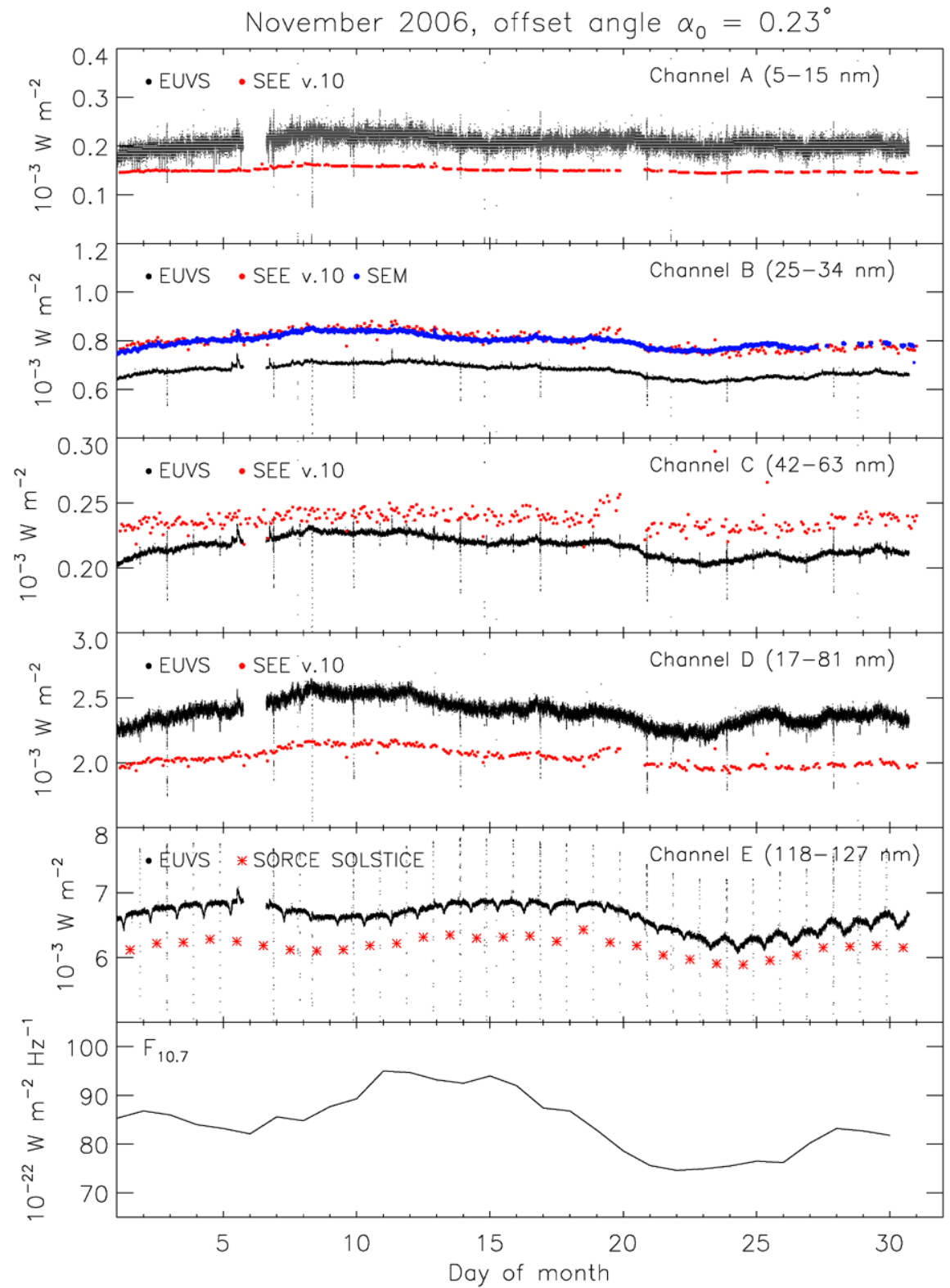

Figure 6 Comparisons for November 2006.

\subsubsection{Energy Flux Differences Among the Sensors}

The discussions to follow address all four months even though specific months are generally not singled out. Regarding SEE/EUVS differences to be discussed shortly, it is helpful to recall that their energy fluxes at a given time and for a given channel are obtained from 
Table 5 Max-to-min EUVS energy flux ratios over four solar rotations. Within a given channel, all energy fluxes are derived using a single spectral shape. The impact of this constraint on the ratios should be insignificant over the four months of quiescent solar activity.

\begin{tabular}{llllll}
\hline $\begin{array}{l}\text { Solar } \\
\text { Rotation }\end{array}$ & \multicolumn{6}{l}{ EUVS $Q_{\max } / Q_{\min }$ by channel } & & & \\
\cline { 2 - 6 } & $\mathrm{A}$ & $\mathrm{B}$ & $\mathrm{C}$ & $\mathrm{D}$ & $\mathrm{E}$ \\
\hline $1^{*}$ & 1.05 & 1.06 & 1.05 & 1.05 & 1.03 \\
$2^{*}$ & 1.25 & 1.17 & 1.16 & 1.18 & 1.07 \\
$3^{*}$ & 1.20 & 1.15 & 1.14 & 1.16 & 1.07 \\
$4^{*}$ & 1.09 & 1.09 & 1.08 & 1.08 & 1.07 \\
\hline
\end{tabular}

* Rotation 1: August; Rotation 2: September; Rotation 3: late September to mid-October; Rotation 4: midOctober into November.

Equation (5) where the sum is over either an EUVS or a SEE spectrum. The spectra differ primarily in magnitude assuming that the 1 July SEE spectrum (used to process EUVS count rates) has the same shapes within channel boundaries as other SEE spectra over the four months. There is support for this, given the generally good agreement in relative variability between EUVS and SEE over these months. A consequence of similar spectral shapes for a given channel is an essentially fixed percent difference in energy flux values, regardless of the selected reporting interval. We have Channel $\mathrm{C}$ in mind, where the poor choice of interval $(42-63 \mathrm{~nm})$ has no impact on the relative difference between EUVS and SEE energy fluxes, i.e., the same relative difference will be seen if the interval is changed to, say, 17-67 nm where the full EUVS signal resides. Other examples are Channels A and B, where the same relative EUVS/SEE differences occur with the reporting intervals extended to shorter wavelengths. It becomes apparent from the above comments that the issues raised in Appendix C about the impact on EUVS energy fluxes from changes in spectral shapes, while important, do not play a role in analyzing EUVS/SEE differences. Thus, we can turn our attention to other sources of calibration error.

4.2.3.1. Channel A EUVS data for this channel are noisier than for the other channels. The noise is thought to arise from the spacecraft environment as well as the sensor itself. Some of the sensor-related noise is due to the cycling of a heater that keeps the solar sensor suite from getting too cold. In spite of the noise, the overall variation is reasonably well correlated with $F_{10.7}$. There is less variation in the SEE fluxes but there is still overall agreement to $20 \%$ or less. The XPS Level 4 product used in the SEE Level 3 products is based on CHIANTI spectral models scaled to match the signals from the broad-band $(7-10 \mathrm{~nm})$ photometers in XPS (Woods et al., 2008). Some of the differences between EUVS A and XPS data could be related to any missing emission lines in the CHIANTI spectral model that would influence the solar variability in the XPS data product. Additional validation for EUVS A, and resolution of this issue regarding variability of SEE vs. EUVS A, is anticipated with the SDO EUV Variability Experiment (EVE) at $0.1 \mathrm{~nm}$ resolution once SDO observations begin in 2010 (Woods, Lean, and Eparvier, 2006).

4.2.3.2. Channel $B$ The energy flux within the He II $30.4 \mathrm{~nm}$ line is primarily responsible for the behavior seen in this channel. There is reasonably good correlation with $\mathrm{F}_{10.7}$ for each data set. SEE and SEM are $\sim 25 \%$ greater than EUVS in August with only modest changes to these differences during the other months. The rise in the SEE irradiances near 16 September 2006 is currently thought to be due to a problem with some of the instrumental 
corrections for the SEE EGS data. The agreement between SEE and SEM argues in favor of a calibration adjustment to EUVS. Any such adjustment will need to come from instrument functions or calibration factors (particularly, detector response and $B_{n}$ and $G_{n}$ ) rather than a change in assumed spectral shape (see text just prior to Section 4.2.3.1). Care must be taken if adjustments are made to the grating function since it is common to Channels A and B. Further investigation of the discrepancies in the data is called for.

4.2.3.3. Channel $C$ As with Channel $\mathrm{B}$, there is reasonably good agreement in relative variability between EUVS and SEE over the four months. SEE energy fluxes are $\sim 15-20 \%$ larger than EUVS values, independent of the chosen reporting interval. If one is interested in mapping either SEE or EUVS values from the current interval $(42-63 \mathrm{~nm})$ to that containing the full EUVS signal $(17-67 \mathrm{~nm})$, the needed scaling factor is $\sim 8.0$ based on the applied spectral shape from the 1 July SEE spectrum.

4.2.3.4. Channel $D$ SEE energy fluxes are 10-15\% less than EUVS values in August with the difference increasing to $15-20 \%$ by November. The relative variation with time for EUVS is similar to its behavior for the previous channels just discussed. SEE closely matches EUVS variability in August but is noticeably flatter in the first half of September, which calls for further investigation. Compared to September, there is better tracking of EUVS variability in October and November but not as good as in August. We recall from the discussion after Equation (5) that only $\sim 1 \%$ of the Channel D signal comes from wavelengths longward of $50 \mathrm{~nm}$ and that the accuracy of reporting from 17 to $81 \mathrm{~nm}$ instead of 17 to $50 \mathrm{~nm}$ depends on the relative accuracy between the spectral shapes to either side of $50 \mathrm{~nm}$ making up the full spectral segment needed to derive the Channel D conversion factor. This accuracy issue does not arise with regard to the relative error between EUVS and SEE, since the same spectral shape applies to both and in turn keeps the error constant regardless of reporting interval.

4.2.3.5. Channel E HI Ly $\alpha$ dominates the signal for this channel (more than He II $30.4 \mathrm{~nm}$ in Channel B) and, consequently, as discussed in Appendix C, the processed data are not sensitive to the underlying spectral shape. Here, we have switched to SORCE SOLSTICE daily Ly $\alpha$ measurements, which have a higher signal-to-noise ratio. There is good agreement in variability between the two sets of measurements with generally less than $10 \%$ differences in absolute magnitudes.

\subsection{December 2006 X9 flare}

Very limited useful EUVS data are available in December due to PLT activities but the sensor was locked on the Sun during the X9 flare that began shortly after $10 \mathrm{~h}$ UT on 5 December 2006. Figure 7 shows the progression of the flare in the five EUVS channels and as recorded between 0.1 and $0.8 \mathrm{~nm}$ by the GOES-12 XRS. The EUVS profiles are based on several different spectral characterizations identified in the top panel and further discussed shortly. Channel E profiles that are indistinguishable from one another exclude NRLEUV-based profiles since the NRLEUV spectra being used do not extend to Channel E wavelengths. The asterisks display energy fluxes obtained from spectra based on SORCE XPS measurements. The diamonds are corresponding EUVS values using the available XPS spectral shapes. The LEO orbit from which the XPS measurements were made precluded observations past 10.73 UT during this particular orbit. XPS-based spectra extend to $40 \mathrm{~nm}$ and are thus restricted to Channels A and B for comparisons with EUVS. Further discussion of the comparisons appears below. 


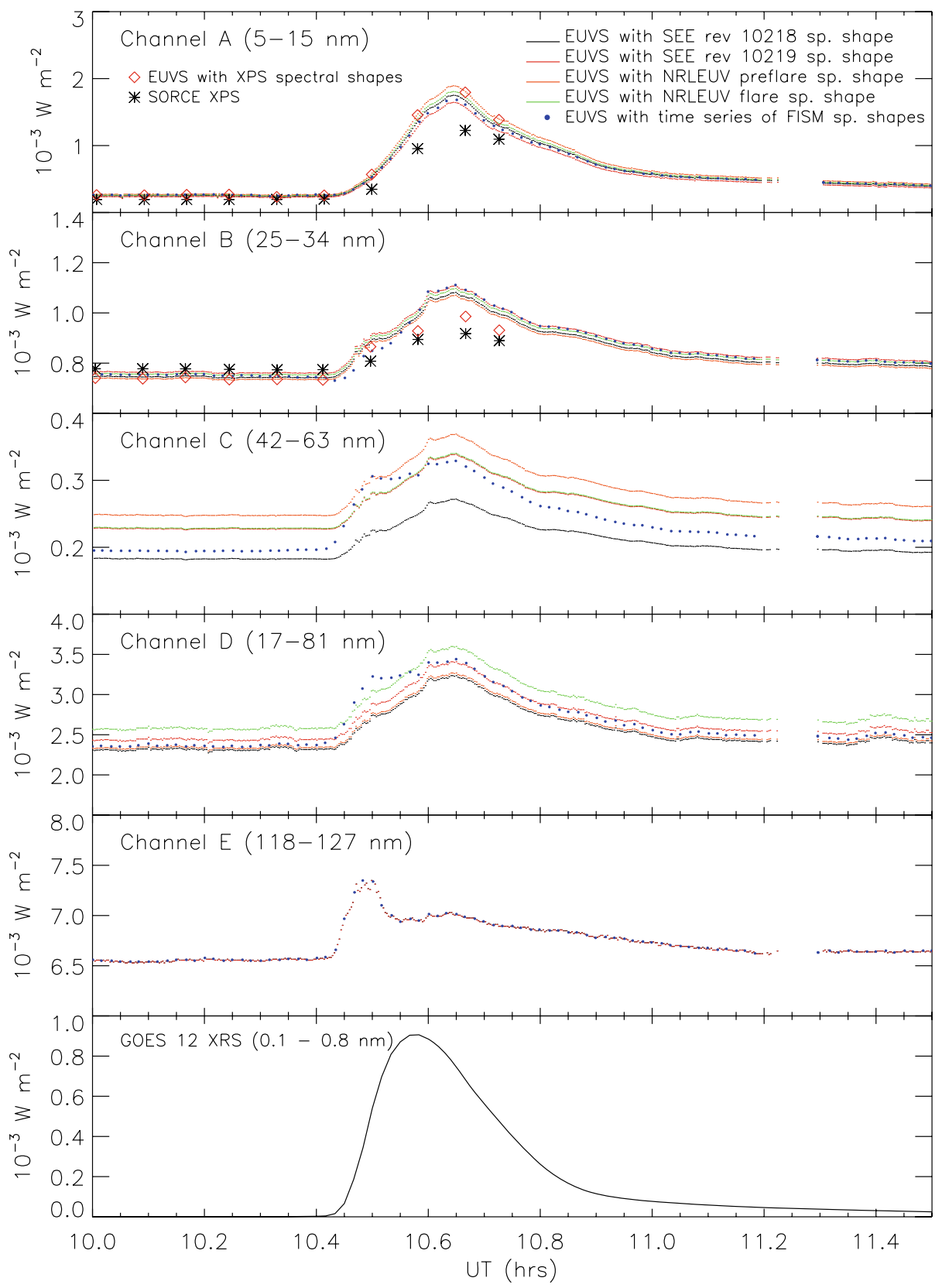

Figure 7 The 5 December 2006 X9 flare recorded by EUVS and SORCE/XPS. The spectral shapes used to derive conversion factors and energy fluxes come from the sources listed in the top panel. They include the SEE spectrum from the 28 October 2003 X-class flare (from orbit 10219 ), a preflare SEE spectrum (from orbit 10218), NRLEUV preflare and flare spectra (but not addressing the same flare), and FISM spectra (see text for details). The bottom panel shows GOES-12 XRS data from 0.1 to $0.8 \mathrm{~nm}$. 
Table 6 Flare and preflare energy fluxes from Figure 7 and their ratios based on spectral shapes from the orbit 10218 SEE spectrum in Figure 20. When using time-independent spectral shapes, the ratios are independent of the spectrum providing these shapes. There are unknown errors in the ratios by constraining the shapes to be time-independent, but some indication of their magnitudes is provided by the spreads among channelspecific curves in Figure 7. Original values to more significant figures were used to calculate the ratios.

\begin{tabular}{llll}
\hline Channel & $\begin{array}{l}\text { Peak energy flux } \\
\left(\mathrm{mW} \mathrm{m}^{-2}\right)\end{array}$ & $\begin{array}{l}\text { Preflare value } \\
\left(\mathrm{mW} \mathrm{m}^{-2}\right)\end{array}$ \\
\hline A & 1.75 & 0.256 & Ratio \\
B & 1.08 & 0.742 & 6.84 \\
C & 0.272 & 0.182 & 1.46 \\
D & 3.24 & 2.31 & 1.49 \\
E & 7.35 & 6.57 & 1.40 \\
\hline
\end{tabular}

The flare began at $~ 10.4 \mathrm{~h} \mathrm{UT}$ and peaked in Channels A $-\mathrm{D}$ at $10.65 \mathrm{~h}$ and in Channel E at $10.48 \mathrm{~h}$. Table 6 shows peak-to-preflare energy flux ratios along with corresponding flux values derived with the use of a single conversion factor per channel based on the SEE preflare spectrum recorded during orbit 10218 . For any given channel, some indication of the error in its ratio due to this restriction may be provided by the spread among its profiles in Figure 7. The ratio reaches 6.8 for Channel A with much weaker responses in the other channels situated at longer wavelengths. This is qualitatively consistent with, e.g., SEE flare observations (see Figure 20 and Woods et al., 2003) and model spectra (see, e.g., Warren, Mariska, and Lean, 2001 and Lean et al., 2003).

The purpose of showing the multiple profiles per channel in Figure 7 is to provide visual displays of some of the differences in $Q$ values shown in Tables 12 and 13. The SEE and NRLEUV spectral characterizations identified in the top panel were introduced through Figures 1 and 20. An EUVS profile has been added for each channel that uses a series of FISM spectral shapes derived specifically for this flare.

The degree of spread going from one channel to the next for Channels A-D has been analyzed in Appendix $\mathrm{C}$ where it is also noted that spectral variability should have essentially no effect on Channel $\mathrm{E}$ due to the dominance by Ly $\alpha$ and only a weak effect on Channel B where He II $30.4 \mathrm{~nm}$ dominates, but to a lesser extent. The large spread for Channel $\mathrm{C}$ is simply an artifact of choosing a poor reporting interval for that channel. As noted in Section 4.1 and Appendix C, the displayed spreads simply reflect changes among a set of selected spectral shapes and do not necessarily reflect expected uncertainties due to insufficient knowledge at this time about the true spectral variability under a wide range of conditions.

There is good preflare agreement between SORCE XPS and EUVS (see top two panels in Figure 7). Near the peak of the flare, the Channel A EUVS value derived with the XPS spectral shape is $\sim 35 \%$ greater than the XPS value with a corresponding difference of less than about $10 \%$ for Channel B. All EUVS values based on the various applied spectral shapes lie within about $15 \%$ and $10 \%$ of one another for Channels A and B, respectively. The cause of the larger XPS/EUVS differences for Channel A remains to be resolved. It is likely associated with the treatment of signals from different spectral regions within a very dynamic range during flares, especially shortward of $5 \mathrm{~nm}$. Resolution may have to await the availability of SDO EVE spectral measurements.

As a final observation, we note the significantly different temporal variation for Ly $\alpha$ compared to the shorter wavelengths (see, e.g., Woods et al., 2003, 2004 for earlier reports of this effect for Ly $\alpha$ ). Its dominant peak occurs just prior to 10.50 UT during the impulsive 
phase of the flare and its gradual phase is less dominant with a peak at 10.65 UT. This impulsive phase structure is also present in Channels $\mathrm{B}-\mathrm{D}$ but as shoulders prior to the gradual phase peak at 10.65 UT. This is typical behavior for flare variations for soft X-ray emissions (see, e.g., Neupert, 1968). It is interesting to see that the time-dependent FISM-based EUVS impulsive phase values for Channels $\mathrm{C}$ and $\mathrm{D}$ show stronger profile enhancements compared to the remaining profiles but that the opposite is true for Channel $\mathrm{B}$.

\subsection{Constructing EUVS-Based Spectra from 1 to $80 \mathrm{~nm}$}

The basis for constructing EUVS-based spectra comes from Equation (4), which gives the absolute magnitude of a channel-specific spectral segment based on the measured counts for that channel (examples of segments for Channels A-D appear in the bottom panels of Figures 16-19). Ideally, when a spectrum is constructed from such segments, there are no differences among segments where they overlap. The causes of any such differences are deviations from the true behavior in spectral shapes, response functions, calibration factors appearing in Equation (1), or some combination of the three. Consider the situation where the assumed spectral shapes match the true shapes. In other words, the assumed spectrum over the full signal range (here, from 1 to $80 \mathrm{~nm}$ ) is the same as the actual spectrum producing the measured EUVS counts. We can then calculate the expected counts for each segment or channel with the aid of response functions like those given in Figure 2. Interest here is in ratios of counts among the channels so that the absolute magnitude of the full spectrum is not relevant. When EUVS now records its counts, a comparison can be made with the calculated ratios. In spite of there being no errors in the applied spectral shapes, one would still expect differences since the response functions will not perfectly match EUVS's true values along with possible errors in the constants in Equation (1). The same argument applies to the use of true response functions and true values in the constants in Equation (1) but with applied spectral shapes that deviate from their true counterparts. An indication of overall errors comes from the extent of the differences in segments where they overlap. This is illustrated below.

The basis of this exercise is sets of EUVS counts for Channels A-D recorded for low activity in early July and during the 5 December flare. Since we are not including Channel E counts, the spectral range stops at $80 \mathrm{~nm}$ (recall, however, that $99 \%$ of the signal for Channel D occurs shortward of $50 \mathrm{~nm}$ ). There is no inherent obstacle to extending the range to beyond Ly $\alpha$ using Channel E counts. One or both of the Channel D and E segments must then be extended to achieve overlap. It should be kept in mind that there is effectively no signal between 50 and $121 \mathrm{~nm}$ and that we must then fully rely on one EUV model or another to produce extrapolations from both sides of this region (an extrapolation in the direction of longer wavelengths is already being done from 50 to $80 \mathrm{~nm}$ ).

Examples of overlapping channel segments appear in Figure 8 for the set of low activity counts from Table 12. The scaled segments in the top panel utilize SEE spectral measurements during TIMED orbit 10218 (the spectrum is given in Figure 20). This is a non-flare spectrum whose structure leads to small differences in overlap regions compared to overall variability. This is not the case in the bottom panel (for the same set of counts) that uses spectral behavior taken from SEE measurements near the peak of an X28 flare that occurred during the next orbit. Here, there are significant differences between the Channel A and B segments (in general, comments about Channel B apply as well to Channel C) where the segments overlap (only in magnitude since all have the same relative behavior). One can compare spectral behavior between orbits 10218 and 10219 within Figure 8 but more easily using Figure 20. There are no significant differences with decreasing wavelength until 

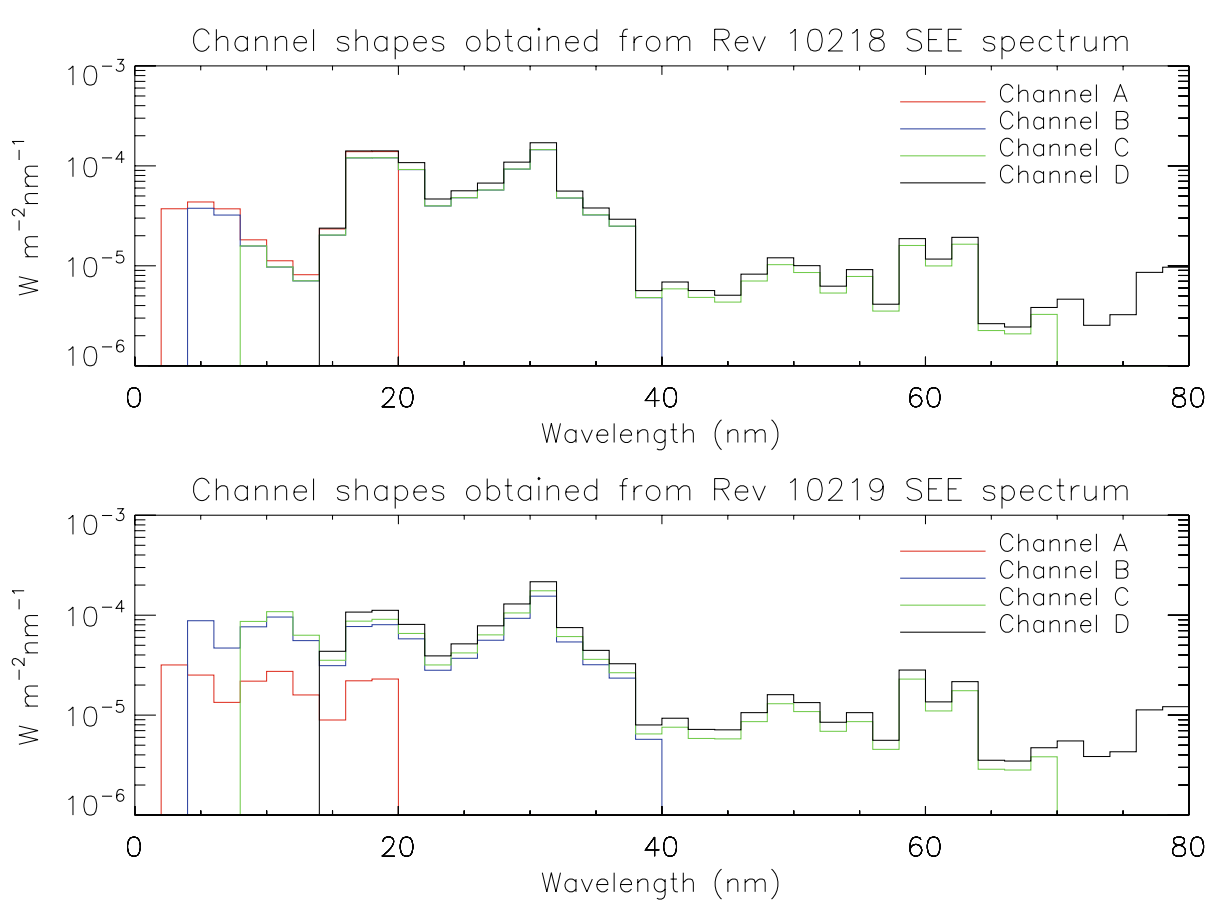

Figure 8 Overlapping spectral segments for Channels A-D are scaled by non-flare EUVS counts listed in Table 12. The top panel shows the segments based on the use of the preflare SEE spectrum from orbit 10218 for converting counts to $\mathrm{W} \mathrm{m}^{-2} \mathrm{~nm}^{-1}$. The segments in the bottom panel are based on the SEE flare spectrum from orbit 10219 and show the incompatibility of the SEE flare spectral shape at short wavelengths with the applied non-flare EUVS data.

reaching $\sim 16 \mathrm{~nm}$ after which the flare spectrum is much flatter. It is interesting to note that $Q$ values for either Channel A or B are similar over their reporting intervals using the different spectral characterizations. A close comparison between panels in Figure 8 for the Channel A segments shows them to be of similar magnitude after accounting for differences in spectral shape. In other words, there is consistency with their $Q$ values being of similar magnitude. Unlike Channel A, the magnitudes of the Channel B and $\mathrm{C}$ segments in the regions of overlap with Channel $\mathrm{A}$ are not determined by counts from here, but rather by counts in the vicinity of He II $30.4 \mathrm{~nm}$. The resulting scaling by counts outside of the overlap region leads to excessive energy flux within the overlap region due to using the inappropriate flat spectral behavior.

One should expect the trend in Figure 8 to reverse by using the above mentioned EUVS flare measurements from 5 December. The effect is shown in Figure 9 for the same spectral shapes. The counts, as taken from the peak of the flare, are 27755, 24211, 24195 , and 27555 for Channels A-D, respectively. The best agreement among segments shortward of $20 \mathrm{~nm}$ is now in the bottom panel where SEE flare spectral behavior is more consistent with the EUVS flare measurements.

We have produced a non-flare spectrum from the segments in the top panel of Figure 8 along with a flare spectrum from the segments in the bottom panel of Figure 9. These appear in Figure 10 where they are over-plotted for easy comparison. Their labels identify the dates, times, and background solar activity levels as represented by the $F_{10.7}$ proxy. Between 4 and 

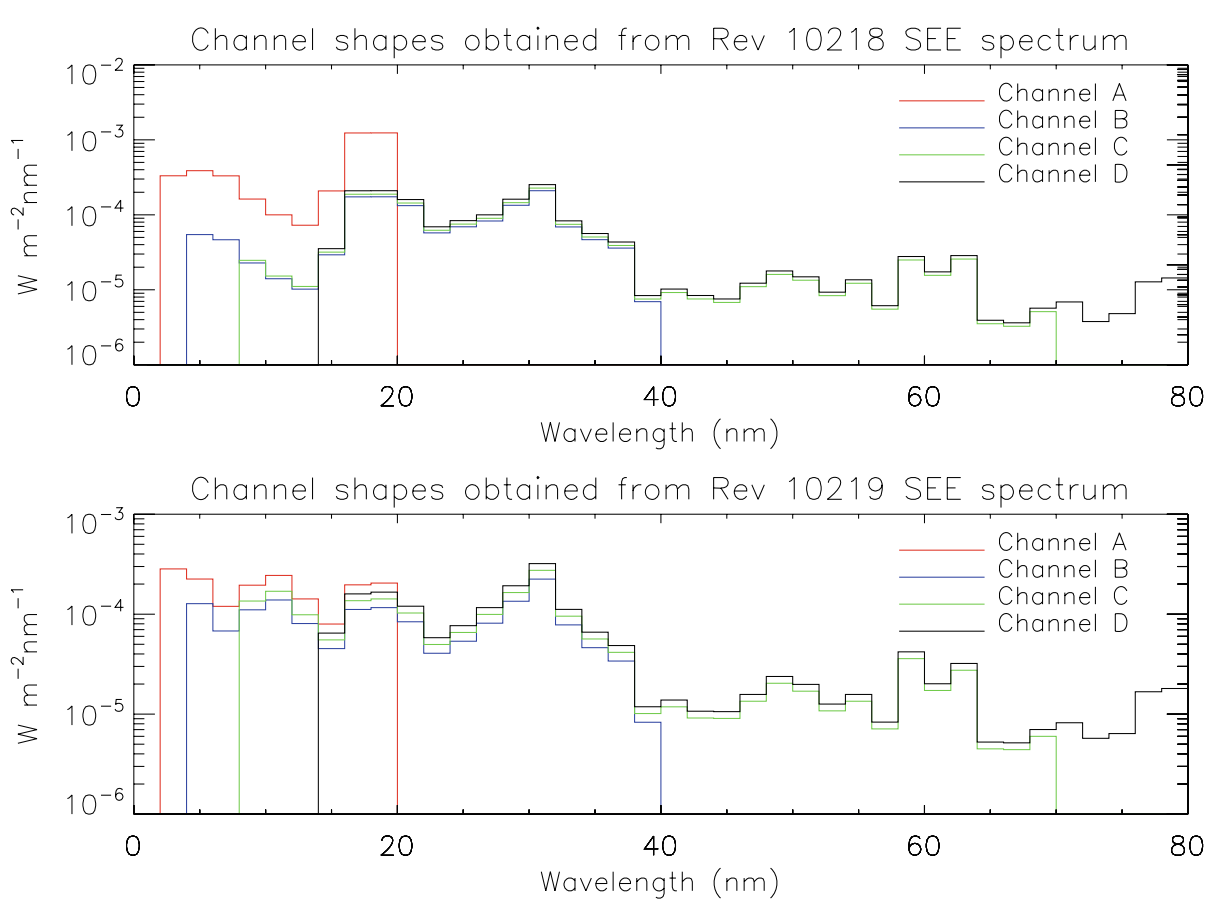

Figure 9 Similar to Figure 8 except for the use of EUVS counts recorded at the peak of the 5 December flare. The trend is seen to reverse with better agreement now in the bottom panel where the flare representation of spectral behavior matches up reasonably well with EUVS flare measurements in contrast to the mismatch in the bottom panel of Figure 8 .

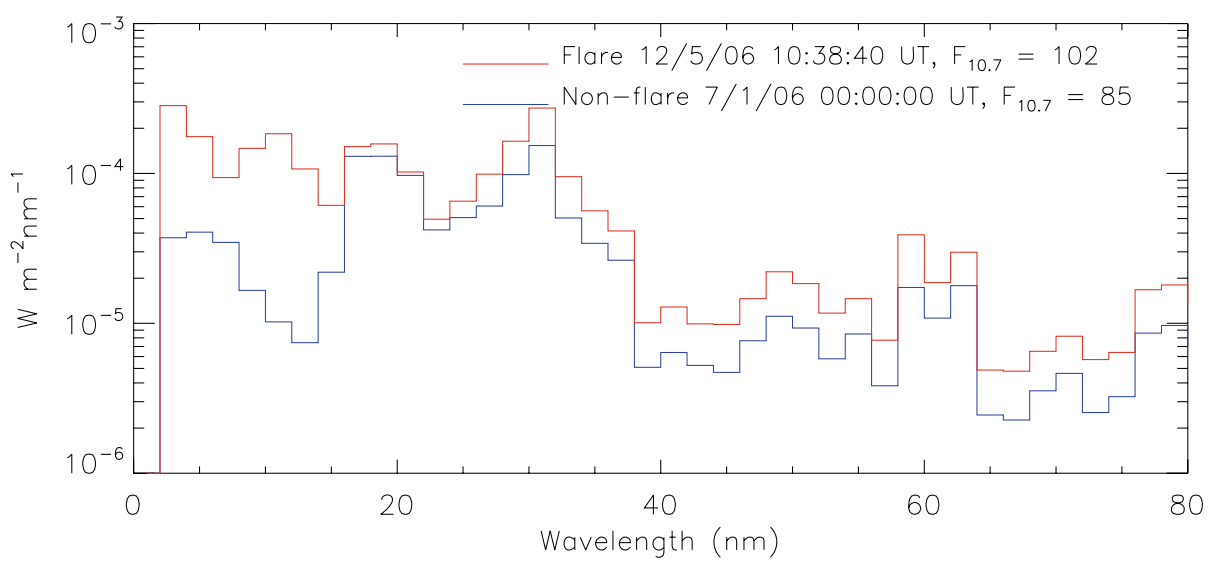

Figure 10 Spectra derived by averaging segments in upper panel of Figure 8 and lower panel of Figure 9.

$70 \mathrm{~nm}$, the spectra were obtained by averaging the overlapping segments. Outside of this region, the values are taken directly from the top and bottom panels in Figures 8 and 9, respectively. Keeping in mind that SEE spectra provided the shapes to the segments in Figures 8 and 9 , the overall shapes of the new spectra, while very similar to those of the SEE spec- 
Table 7 Pre-averaged energy fluxes obtained from channel spectra in Figure 8 (upper panel) and Figure 9 (lower panel). Also included are averaged values obtained from the spectra in Figure 10. Note that here the spectral shapes for deriving the flare energy fluxes are from the orbit 10219 SEE spectrum whereas in Table 6, the orbit 10218 spectrum is used. Also note that the non-flare counts come from 1 July, whereas the counts for the preflare fluxes in Table 6 come from just before the flare. Original values to more significant figures were used to calculate the percentages.

\begin{tabular}{|c|c|c|c|c|c|c|}
\hline \multirow[b]{2}{*}{ Channel } & \multicolumn{3}{|l|}{ Non-flare } & \multicolumn{3}{|l|}{ Flare } \\
\hline & $\begin{array}{l}Q_{n} \text { before } \\
\text { averaging }\end{array}$ & $\begin{array}{l}Q_{n} \text { after } \\
\text { averaging }\end{array}$ & Change & $\begin{array}{l}Q_{n} \text { before } \\
\text { averaging }\end{array}$ & $\begin{array}{l}Q_{n} \text { after } \\
\text { averaging }\end{array}$ & Change \\
\hline A & 0.196 & 0.182 & $-7.5 \%$ & 1.65 & 1.25 & $-24 \%$ \\
\hline B & 0.746 & 0.791 & $+6.0 \%$ & 1.11 & 1.35 & $+22 \%$ \\
\hline $\mathrm{C}$ & 0.174 & 0.188 & $+8.6 \%$ & 0.338 & 0.367 & $+8.5 \%$ \\
\hline $\mathrm{D}$ & 2.18 & 1.99 & $-8.8 \%$ & 3.41 & 3.01 & $-12 \%$ \\
\hline
\end{tabular}

tra, have become modified by averaging in the overlap regions. The modifications amount to magnitude shifts at wavelengths where the number of overlapping segments change and thus preserve SEE spectral behavior except for these shifts. The overall magnitudes of the new spectra, however, are determined by EUVS counts and the assumed response functions in addition to SEE spectral shapes. The percentage of change in $Q$ values arising from averaging may be seen in Table 7. For both the non-flare and flare measurements, channel-specific $Q$ values over their reporting intervals are shown before and after averaging along with the changes. The largest change is a decrease of $24 \%$ for Channel A during the flare that arises from the smaller flux values for Channels B-D shortward of $20 \mathrm{~nm}$ where there is little contribution to their respective signals. This illustrates the point that better spectral characterization under flare conditions is needed shortward of where there is significant signal for these channels (shortward of the $30.4 \mathrm{~nm}$ region). It would seem appropriate to place less weight on Channels B $-\mathrm{C}$ when averaging to obtain the Channel A $Q$ value.

Given the modest differences between segments in overlapping regions under non-flare conditions, there is no strong motivation to develop a more detailed method for producing EUVS-based spectra. More attention is justified under flare conditions at short wavelengths, either weighted averaging or investigations into spectral shape improvements.

\section{Summary}

The reported work provides the first critical examination of GOES-13 EUVS performance, specifically for data recorded during a six-month post-test-launch (PLT) period. This EUVS and its successors on future GOES missions are expected to play a significant role in addressing short-term to long-term thermospheric and ionospheric variability. Nearly continuous observations from August through November 2006 are reported along with those from an X9 flare that occurred on 5 December 2006. These are in the form of energy fluxes (in $\mathrm{W} \mathrm{m}^{-2}$ ) within five spectral channels spanning the EUV region from 1 to $130 \mathrm{~nm}$ at a per-channel sampling rate of about once per $11 \mathrm{~s}$. Considerable attention is given to a newly-developed EUVS calibration model. It is described in Section 3 with details given in Appendix B. Its application to sensitivity studies is also described in Appendix B as well as in Appendix C. A key to the assessment of PLT EUVS data is how they compare with independent measurements. These are taken from SOHO/SEM, TIMED/SEE (Version 10.02), SORCE/XPS, and SORCE/SOLSTICE. The comparisons can be seen in Figures 3-6 and show satisfactory 
overall agreement within expected uncertainties among the various sensors. The following list provides more details with regard to the calibration model, its application to sensitivity studies, and EUVS performance.

- The calibration model derives conversion factors over the wavelength intervals shown in the middle column of Table 1, which in turn are used to convert counts to energy fluxes over these intervals (Equation (1)). For each of the five EUVS channels, the energy flux along with the assumed spectral shape then allows one to specify the flux over what we call the reporting interval (the right column in Table 1). It was noted in Section 4.1 and in Appendix $C$ that a revision to Channel C's reporting interval is in order since most of the signal occurs shortward of this region. Other comments about the intervals for Channels A, B, and D are given in Section 3 after Equation (5).

- The model provides conversion factors and resulting energy fluxes as functions of pointing offsets, cross-disk radiance distributions, and spectral shapes. Appendices B and C illustrate the sensitivity to these parameters. A significant finding is the minimal impact to measurement uncertainties arising from changes to radiance distributions. The greatest source of uncertainty is expected to arise from current lack of knowledge in spectral variability, especially during flares. A detailed discussion on this topic is given in Appendix C.

- The four-panel figures in Appendix C are key to understanding the dependence of energy fluxes on spectral variability. Noteworthy is the third panel in each of these figures, which lists conversion factors and displays their integrands, namely products of the normalized spectral segments and response functions for a given channel. Differences among conversion factors can be clearly understood by comparing the integrands within the panel. It is clear from panels 2 and 3 in these figures that the channel response function alone does not appropriately define the bandpass for the solar measurement, but that the inclusion of a normalized solar spectral segment is needed to define the "true" bandpass for each channel.

- EUVS data for Channels A-D are compared with TIMED/SEE from August through November 2006 (Figures 3 -6). The Channel B comparisons include SOHO/SEM data as well. For Channel E (Ly $\alpha$ ), comparisons are made with data from SORCE/SOLSTICE. The relative differences observed between EUVS and SEE are the same, regardless of the reporting interval. This is a consequence of using SEE spectral shapes to derive conversion factors. SEE energy fluxes are greater than those of EUVS for Channels B and C ( $\sim 25 \%$ and $20 \%$, respectively) and less for Channels A, D, and E ( 20\%, 15\%, and $10 \%$, respectively). SEM energy fluxes are similar to SEE values and are thus also $\sim 25 \%$ greater than EUVS. This difference could be reduced with adjustments to the EUVS transmission grating function (but would also alter Channel A fluxes since the grating is common to both channels) or through adjustments to the constants in Equation (1).

- During the 5 December X9 flare, Figure 7 shows the increase in energy fluxes for all channels with the largest, as expected, occurring for Channel A (by a factor of 6.8). The spread among the multiple profiles displayed for each of the channels reflects spectral sensitivities given in Tables 12 and 13. The panels for Channels A and B also contain values obtained by integrating SORCE/XPS-based spectra over the reporting intervals of these channels and corresponding EUVS values using XPS spectral shapes. Using these shapes, EUVS agrees with XPS to $\sim 10 \%$ or less for Channel B prior to and during the flare. For Channel A, good agreement is also observed prior to the flare but EUVS values then exceed those of XPS by up to $\sim 35 \%$. This behavior is suggested to arise from the different sensitivities shortward of $5 \mathrm{~nm}$ but currently remains unresolved. 
- An important step to constructing spectra from EUVS measurements is presented in Section 4.4. The 2-80 $\mathrm{nm}$ region is addressed, from which full signals are received for Channels A-D. Figure 8 shows overlapping spectral segments, each for a given channel obtained from Equation (4) using the counts for that channel. The emphasis is on absolute differences between segments in the overlapping regions. The recorded counts for the four channels come from a period of low activity. The differences are small compared to overall variability versus wavelength when using a non-flare spectrum for providing segment shapes (consistent with the use of non-flare count values). A much larger difference, on the other hand, occurs between Channel A and B segments using a flare spectrum. The reverse occurs in Figure 9 where a set of counts is taken from the peak of the 5 December flare (a much larger difference using a non-flare spectrum). This interesting exercise demonstrates a technique for assessing the appropriateness of the assumed spectral behavior at short wavelengths (shortward of $20 \mathrm{~nm}$ ) for a given set of EUVS measurements. The non-flare segments in the upper panel of Figure 8 and the flare segments in the bottom panel of Figure 9 have been averaged to produce the spectra shown in a single panel in Figure 10. The argument is made that further refinements under non-flare conditions are probably unnecessary in light of modest differences in overlapping regions that arise from multiple error sources. Further investigation would be worthwhile for intense flares in constructing spectra over the Channel A region.

- Recommended extensions of the reported work include a better quantification of uncertainties (especially with regard to assumed spectral behavior) and further attention to the derivation of flare spectra addressed in the previous bullet. Future spectral measurements by SDO's EVE should be instrumental in reducing errors arising from an incorrectly assumed short wavelength spectral behavior.

Acknowledgements Funding was provided by NOAA's Space Weather Prediction Center through a Memorandum of Understanding with the Naval Research Laboratory. A portion of this funding was directed to the work being reported and provided support to the CPI, SSRC, and NRL personnel on the author list. We thank Phillip Chamberlin for making FISM spectra available for use in our flare analysis.

Open Access This article is distributed under the terms of the Creative Commons Attribution Noncommercial License which permits any noncommercial use, distribution, and reproduction in any medium, provided the original author(s) and source are credited.

\section{Appendix A: Terms and Units}

Irradiance Radiation of the Sun integrated over the full disk and expressed in SI units of power through a unit area (here $\mathrm{W} \mathrm{m}^{-2}$ )

Radiance Similar to irradiance but from a portion of the solar disk

Terms directly related to solar irradiance that are not channel specific

$\lambda \quad$ Wavelength (nm)

$\delta \lambda \quad$ Resolution for calculating response functions (1 $\mathrm{nm}$ for results being reported)

$\phi(\lambda) \quad$ Irradiance spectrum in $\mathrm{W} \mathrm{m}^{-2} \mathrm{~nm}^{-1}$

\section{Terms related to geometry}

$L \quad$ Distance from transmission grating to detector ( $\mathrm{mm}$ )

$d_{i}$ Arc-distance from optical axis $\left(\alpha_{\mathrm{o}}=\alpha=0^{\circ}\right)$ to inner edge of active area of detector (mm)

$d_{\mathrm{o}}$ Arc-distance to outer edge of active area of detector $(\mathrm{mm})$ 
$\alpha_{\mathrm{o}}$ Offset of solar disk from center of field-of-view $\left({ }^{\circ}\right)$

$\alpha \quad$ Solar disk angle $\left(0^{\circ}\right.$ at disk center; $\pm 0.26675^{\circ}$ at edges $)$

$\beta \quad$ Diffraction angle ( \pm for \pm orders)

$m$ Order within diffraction pattern

$p \quad$ Grating period (distance from gap to gap; $\mathrm{nm}$ )

Remaining terms are channel specific but channel index not being displayed

\begin{tabular}{|c|c|}
\hline$\tilde{f}_{\text {det }}(\lambda)$ & Detector function ( $\mathrm{e}^{-} /$photon $)$ \\
\hline$f_{\text {det }}(\lambda)$ & Detector function $\left(\mathrm{A} /\left[\mathrm{W} \mathrm{m}^{-2}\right]\right)$ where A refers to amps \\
\hline$f_{\text {filter }}(\lambda)$ & Filter function (dimensionless) \\
\hline$f_{\text {grating }}(m, \lambda)$ & Transmission grating function (dimensionless) \\
\hline$f_{\text {geom }}\left(m, \lambda, \alpha_{\mathrm{o}}\right)$ & $\begin{array}{l}\text { Geometry function (dimensionless). Defined to be fraction of energy } \\
\text { entering instrument from full solar disk that reaches active area of detec }\end{array}$ \\
\hline$f_{\mathrm{AR}}\left(\lambda, \alpha_{\mathrm{o}}\right)$ & $\begin{array}{l}\text { Absolute response function }\left(\mathrm{A} /\left[\mathrm{W} \mathrm{m}^{-2}\right]\right) \text { for the number of orders con } \\
\text { ered }\end{array}$ \\
\hline$f_{\mathrm{IR}}\left(\lambda, \alpha_{\mathrm{o}}\right)$ & Integrated response function $\left(\mathrm{A} /\left[\mathrm{W} \mathrm{m}^{-2} \mathrm{~nm}^{-1}\right]\right)$ \\
\hline$\phi_{N}(\lambda)$ & $\begin{array}{l}\text { Normalized spectrum in } \mathrm{nm}^{-1} \text {. Normalization is over wavelength inte } \\
\text { specific to a given channel. }\end{array}$ \\
\hline$C$ & Conversion factor $\left(\mathrm{A} /\left[\mathrm{W} \mathrm{m}^{-2}\right]\right)$ \\
\hline \multicolumn{2}{|c|}{$\begin{array}{ll}\lambda_{\min }, \lambda_{\max } & \text { Defines the interval over which } \phi_{N} \text { and } f_{\mathrm{IR}} \text { are specified in order to calcu- } \\
\text { late } C\end{array}$} \\
\hline$\Delta \lambda$ & $\begin{array}{l}\text {-specified channel width whose limits should not fall outside of the } \\
\lambda_{\max } \text { interval }\end{array}$ \\
\hline
\end{tabular}

\section{Appendix B: Geometry Functions and Sensitivity of Conversion Factors to Pointing Offsets and to Cross-Disk Radiance Distributions}

\section{B.1. Sensor Schematics and Sign Conventions}

Figure 11 provides a simple schematic showing the placements of the Channel A and B detectors along with their common transmission grating. We shall refer to this as a top view with detector A on the right. The coordinates $x$ and $y$ (not shown) are used to designate distances across and perpendicular to the page, respectively. The line bisecting the diagram is the optical axis with negative (positive) $x$ values to the right (left). The coordinate $y$ is used to specify vertical distances of the aperture and detectors. It plays no role in the following discussion, since the vertical extents of the detectors are sufficient to capture the vertical extent of the solar disk. There is a similar schematic for Channels $\mathrm{C}$ and $\mathrm{D}$ with detector $\mathrm{C}$ on the right. Channel E possesses its own grating with its detector also on the right. Figure 11 identifies several key variables. Starting at the top is $\alpha_{0}$, the offset angle of the solar disk within the EUVS field-of-view (FOV) $\left(0^{\circ}\right.$ in the schematic) that increases from right to left. The angle $\alpha$ is a solar disk angle $\left(0^{\circ}\right.$ at disk center) and, like $\alpha_{\mathrm{o}}$, increases from right to left. The line at angle $\alpha$ from the solar disk to the grating represents the radiance from a vertical slice of the disk. A portion becomes diffracted through angle $-\theta^{\mathrm{A}}$ (for some negative order and wavelength) that lands on detector A. Another portion becomes diffracted through angle $\theta^{\mathrm{B}}$ (a positive order) that lands on detector $\mathrm{B}$. The sign and magnitude of a given diffraction angle are provided by the grating equation to be presented shortly. Remaining variables in the diagram are $L^{\mathrm{A}}$ and $L^{\mathrm{B}}$, the distances from the grating to detectors $\mathrm{A}$ and $\mathrm{B}$ and the arc lengths $-d_{i}^{\mathrm{A}}$ and $d_{i}^{\mathrm{B}}\left(-d_{\mathrm{o}}^{\mathrm{A}}\right.$ and $\left.d_{\mathrm{o}}^{\mathrm{B}}\right)$ from the optical axis to the nearest (farthest) edges of the detectors. 
Figure 11 Schematic showing the placement of the channel A and $\mathrm{B}$ detectors along with their common transmission grating. Also identified are several key variables including $\alpha_{\mathrm{o}}$, the offset angle of the solar disk within the EUVS field-of-view (FOV) $\left(0^{\circ}\right.$ in the schematic), $L^{\mathrm{A}}$ and $L^{\mathrm{B}}$, the distances from the grating to detectors $\mathrm{A}$ and $\mathrm{B}$, and the arc lengths $-d_{i}^{\mathrm{A}}$ and $d_{i}^{\mathrm{B}}\left(-d_{\mathrm{o}}^{\mathrm{A}}\right.$ and $\left.d_{\mathrm{O}}^{\mathrm{B}}\right)$ from the optical axis to the inner (outer) edges of the detectors.

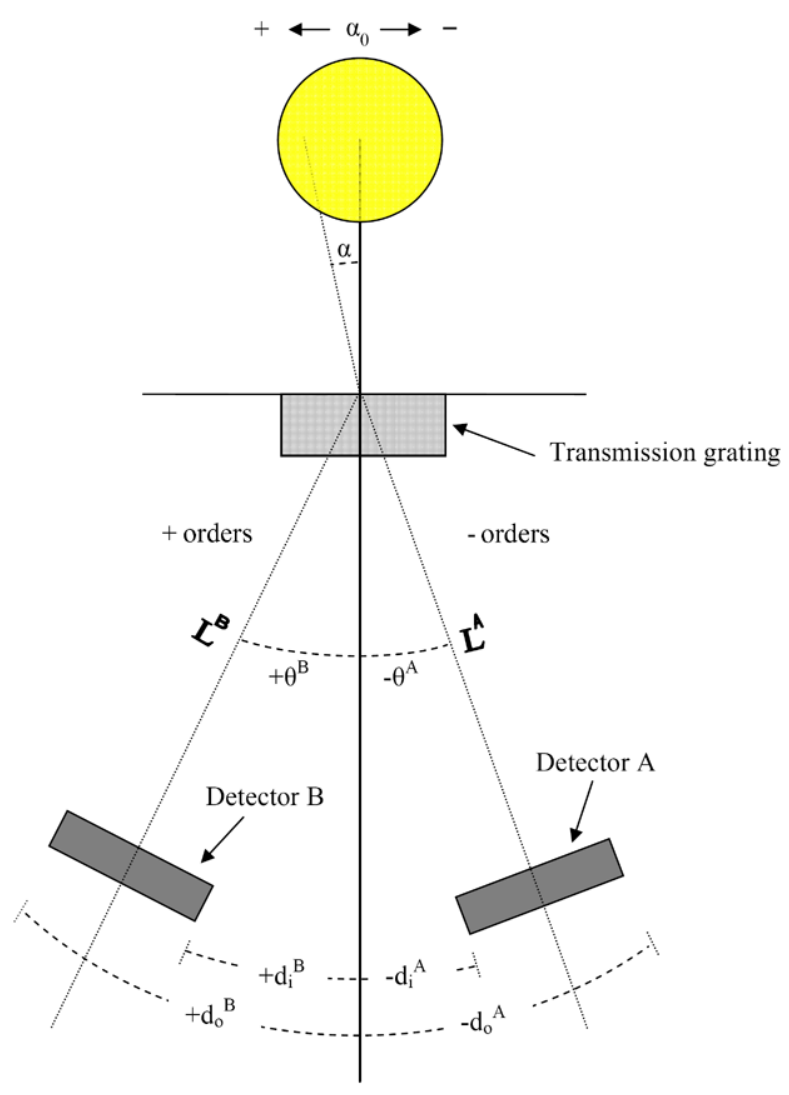

\section{B.2. Grating Equation and Geometry Function}

We define the geometry function, $f_{\text {geom }}(\lambda)$, to be the fraction of the total disk intensity per $\mathrm{nm}$ at $\lambda$ incident on a transmission grating that is diffracted onto the detector. To derive the geometry function, we assume for now that the grating is a perfect transmitter. Imperfect transmission by the grating is formally addressed in the next section. Let $I\left(\lambda, q^{\prime}\right)$ be the source radiance at wavelength $\lambda$ and angle $\theta^{\prime}$ incident on a transmission grating that is diffracted as intensity $I(\lambda, q)$ into a single specific order at wavelength $\lambda$ and angle $\theta$ from the optical axis. The geometry function may be obtained by dividing the total intensity incident on the detector by the total intensity incident on the diffraction grating from the source, i.e.,

$$
f_{\text {geom }}(\lambda)=\frac{\int_{\theta_{i}}^{\theta_{0}} I(\lambda, \theta) \mathrm{d} \theta}{\int_{-\pi}^{\pi} I\left(\lambda, \theta^{\prime}\right) \mathrm{d} \theta^{\prime}}
$$

where $\theta_{i}\left(\theta_{0}\right)$ is the angle in the diffraction plane between the optical axis and the inner (outer) edge of the detector. In Equation (B.1), we assume that the full vertical extent of the source radiation is transmitted by the grating onto the detector. Equation (B.1), therefore, represents the reduction in intensity received by the detector due to the finite size of the detector within the plane of diffraction. To determine the fraction of the total intensity that falls on the detector, we must map the angles $\theta_{i}$ and $\theta_{\mathrm{o}}$ defining the angular extent of the 
Figure 12 Schematic identifying key geometrical variables with positive offset of the solar disk within the EUVS field-of-view $\left(\alpha_{\mathrm{o}}\right)$. These include diffraction angles $\theta_{i}$ and $\theta_{\mathrm{o}}$, radial distance $L$ from grating to the detector center, distances $d_{1}$ from transmission grating to detector center and inner and outer edges of unmasked portion of detector, and distances $d_{2}$ from optical axis to detector center and edges.

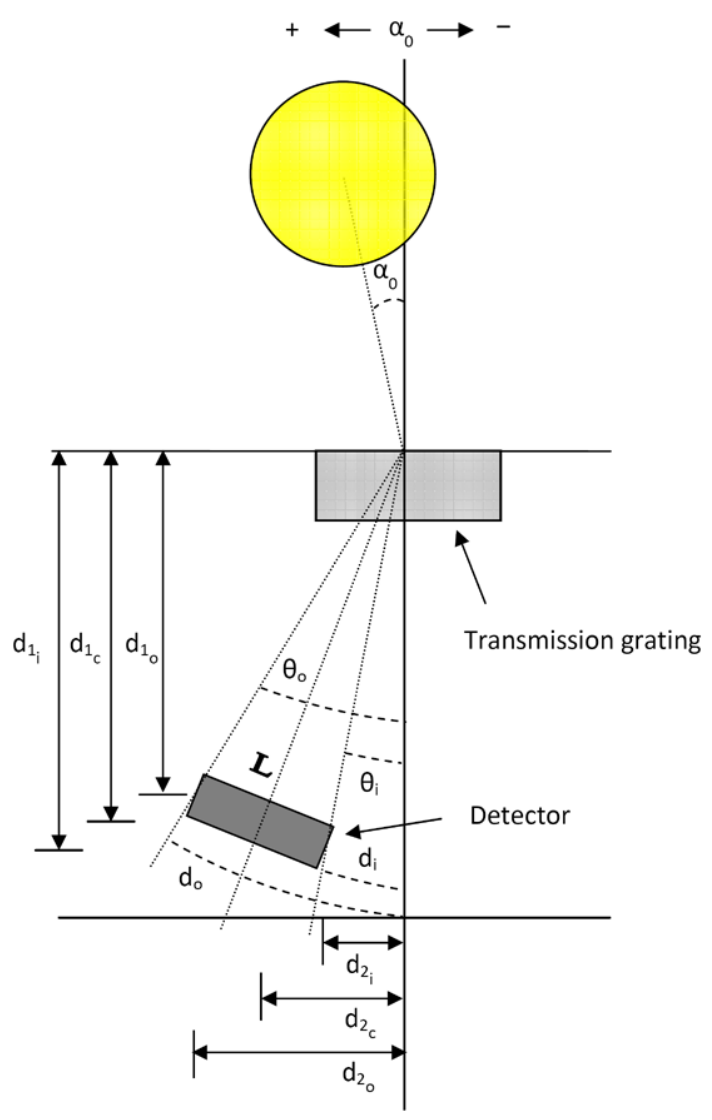

detector in the plane of diffraction back to the source side of the grating. To achieve this, we must make use of the grating equation.

The grating equation provides the relationship between diffraction angle $\theta$ and the angle of incidence $\left(\alpha_{\mathrm{o}}+\theta^{\prime}\right)$ for a given wavelength $\lambda$. Using these variables, the equation has the form

$$
m \lambda=p\left[\sin \left(\alpha_{\mathrm{o}}+\theta^{\prime}\right)+\sin \theta\right]
$$

where $m$ designates the diffraction order and $p$ is the grating period in $\mathrm{nm}$ (distance from gap to gap). The coordinate system used to specify detector dimensions is a Cartesian system co-aligned with the optical axis of the transmission grating. Detectors are located within the measurement plane by specifying distances parallel and perpendicular to the optical axis, as shown in Figure 12. Table 8 gives EUVS detector dimensions in the described coordinate system (provided by SWPC through co-authors RAV and SMH).

In order to properly use the values in Table 8, they must be converted to angular values suitable for use in the grating equation. The coordinate conversion is obtained directly from the geometry depicted in Figure 12 . The quantities $L, d_{i}$, and $d_{\mathrm{o}}$ are given by

$$
L=\sqrt{\left(d_{1_{c}}\right)^{2}+\left(d_{2_{c}}\right)^{2}}
$$


Table 8 Detector placement within the measurement plane.

\begin{tabular}{|c|c|c|c|c|c|c|}
\hline \multirow[b]{2}{*}{ Channel } & \multicolumn{3}{|c|}{$d_{1}$ dimensions $(\mathrm{cm})$} & \multicolumn{3}{|c|}{$d_{2}$ dimensions $(\mathrm{cm})$} \\
\hline & Inner edge & Center & Outer edge & Inner edge & Center & Outer edge \\
\hline A & 16.83 & 16.81 & 16.79 & 0.85 & 1.14 & 1.44 \\
\hline B & 14.15 & 14.10 & 14.05 & 1.76 & 2.09 & 2.41 \\
\hline $\mathrm{C}$ & 17.38 & 17.30 & 17.23 & 1.75 & 2.29 & 2.84 \\
\hline $\mathrm{D}$ & 15.34 & 15.24 & 15.14 & 2.73 & 3.22 & 3.70 \\
\hline $\mathrm{E}$ & 15.28 & 15.24 & 15.21 & 2.98 & 3.15 & 3.33 \\
\hline
\end{tabular}

Table 9 Parameters needed to specify geometry functions.

\begin{tabular}{llllrr}
\hline Channel & $m$ & $p(\mathrm{~nm})$ & $L(\mathrm{~mm})$ & $d_{i}(\mathrm{~mm})$ & $d_{\mathrm{O}}(\mathrm{mm})$ \\
\hline A & -1 to -6 & 200 & 168 & -8.5 & -14.4 \\
B & +1 to +3 & 200 & 142 & 17.7 & 24.2 \\
C & -1 to -4 & 400 & 174 & -17.5 & -28.5 \\
D & +1 to +4 & 400 & 155 & -29.5 & 37.4 \\
E & -1 & 600 & & & -33.5 \\
\hline
\end{tabular}

$$
\begin{aligned}
d_{i} & =L \tan ^{-1}\left(\frac{m d_{2_{i}}}{|m| d_{1_{i}}}\right), \\
d_{\mathrm{o}} & =L \tan ^{-1}\left(\frac{m d_{2_{\mathrm{o}}}}{|m| d_{1_{\mathrm{o}}}}\right) .
\end{aligned}
$$

Table 9 shows $m$ values considered for each channel as well as $p$ values for the three gratings. In addition, values of $L, d_{i}$, and $d_{\mathrm{o}}$ are included that are needed as well for deriving geometry functions.

Using the grating equation, we can now introduce a change of variables for the integration limits given in the numerator of Equation (B.1). To map angles $\theta_{i}, \theta_{\mathrm{o}}$ from the detector side of the grating to the source side, we rearrange Equation (B.2) as

$$
\theta^{\prime}=\sin ^{-1}\left[\frac{m \lambda}{p}-\sin \theta\right]-\alpha_{\mathrm{o}} .
$$

Using Equation (B.4) we can rewrite Equation (B.1) as

$$
f_{\text {geom }}(\lambda)=\frac{\int_{\theta_{i}^{\prime}}^{\theta_{0}^{\prime}} I\left(\lambda, \theta^{\prime}\right) \mathrm{d} \theta^{\prime}}{\int_{-\pi}^{\pi} I\left(\lambda, \theta^{\prime}\right) \mathrm{d} \theta^{\prime}}
$$

where

$$
\begin{aligned}
& \theta_{i}^{\prime}=\sin ^{-1}\left[\frac{m \lambda}{p}-\sin \theta_{i}\right]-\alpha_{\mathrm{o}}, \\
& \theta_{\mathrm{o}}^{\prime}=\sin ^{-1}\left[\frac{m \lambda}{p}-\sin \theta_{\mathrm{o}}\right]-\alpha_{\mathrm{o}} .
\end{aligned}
$$




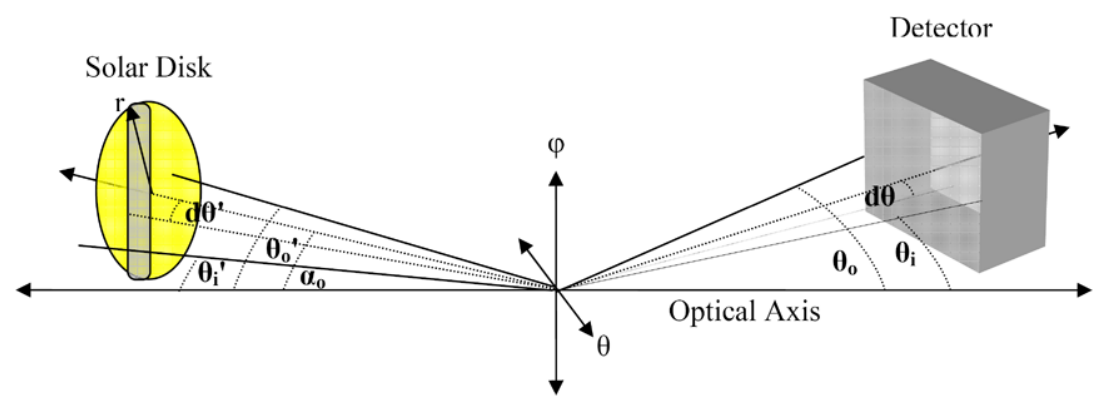

Figure 13 Schematic identifying key angular quantities for deriving a geometry function for uniform radiance across a solar disk of an angular extent given by $2 r$. Angle $\alpha_{0}$ represents an offset of the sun center within the EUVS field-of-view. Diffraction angles $\theta_{i}$ and $\theta_{\mathrm{O}}$ correspond to the inner and outer edges respectively of the unmasked portion of detector from the optical axis. Angles $\theta_{i}^{\prime}$ and $\theta_{\mathrm{o}}^{\prime}$ correspond to the inner and outer edges of the detector mapped to the source side of the grating as determined by the grating equation.

We are now in a position to apply Equations (B.5) and (B.6) to specific characterizations of the source radiance $I\left(\lambda, \theta^{\prime}\right)$.

\section{B.2.1. Uniform Radiance Distribution}

If no knowledge of the spatial distribution of the source radiance is available, some assumptions must be made to derive a geometry function. We begin by assuming an angular extent of $2 r=0.5335^{\circ}$ for the solar disk (this value is used for all calculations being reported and is taken from Sofia, Heaps, and Twigg, 1994) and a center offset of angle $\alpha_{\mathrm{o}}$ from the optical axis as shown in Figure 13. For all EUVS calculations to follow, we have included a correction of the Sun - Earth distance to $1 \mathrm{AU}$. If we next assume a uniform radiance across the disk for all $\lambda^{\prime}$ s, the quotient in Equation (B.5) simplifies to dividing the area of the solar disk between angles $\theta_{i}^{\prime}$ and $\theta_{\mathrm{o}}^{\prime}$ by the total area. Under these assumptions, Equation (B.5) yields

$$
\begin{aligned}
f_{\text {geom }}(\lambda) & =\frac{1}{\pi r^{2}} \int_{\theta_{i}^{\prime}}^{\theta_{\mathrm{o}}^{\prime}} 2 \sqrt{r^{2}-\theta^{\prime 2}} \mathrm{~d} \theta^{\prime} \\
& =\frac{1}{\pi r^{2}}\left[\theta_{\mathrm{o}}^{\prime} \sqrt{r^{2}-\theta_{\mathrm{o}}^{\prime 2}}+r^{2} \sin ^{-1}\left(\frac{\theta_{\mathrm{o}}^{\prime}}{r}\right)-\theta_{i}^{\prime} \sqrt{r^{2}-\theta_{i}^{\prime 2}}+r^{2} \sin ^{-1}\left(\frac{\theta_{i}^{\prime}}{r}\right)\right]
\end{aligned}
$$

where $\theta_{i}^{\prime}$ and $\theta_{\mathrm{o}}^{\prime}$ are given by Equation (B.6).

\section{B.2.2. Radiance Distribution from SXI Image}

Since SXI is co-aligned with EUVS, we can utilize SXI images as a proxy for the spatial distribution of the source radiance with the implicit assumption that a spatial distribution based on X-ray wavelengths is similar to one based on EUV wavelengths. In the present case, we follow an approach similar to that used above and determine the ratio of the total counts in the SXI image between angles $\theta_{i}^{\prime}$ and $\theta_{\mathrm{o}}^{\prime}$ to the total counts in the image. Again, we assume that the full vertical extent of the image is transmitted by the grating onto the detector. A schematic is given in Figure 14, similar to that for uniform radiance addressed in Figure 13. If we let the function $g\left(\theta^{\prime}, \varphi^{\prime}\right)$ represent the number of counts in an SXI image 


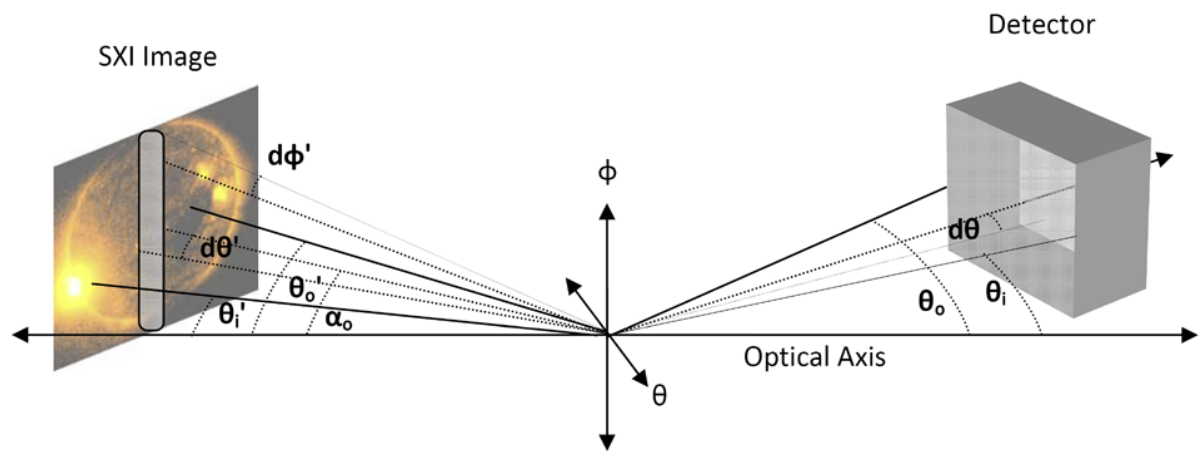

Figure 14 Similar to Figure 13 with the image of uniform cross-disk radiance replaced by an SXI image.

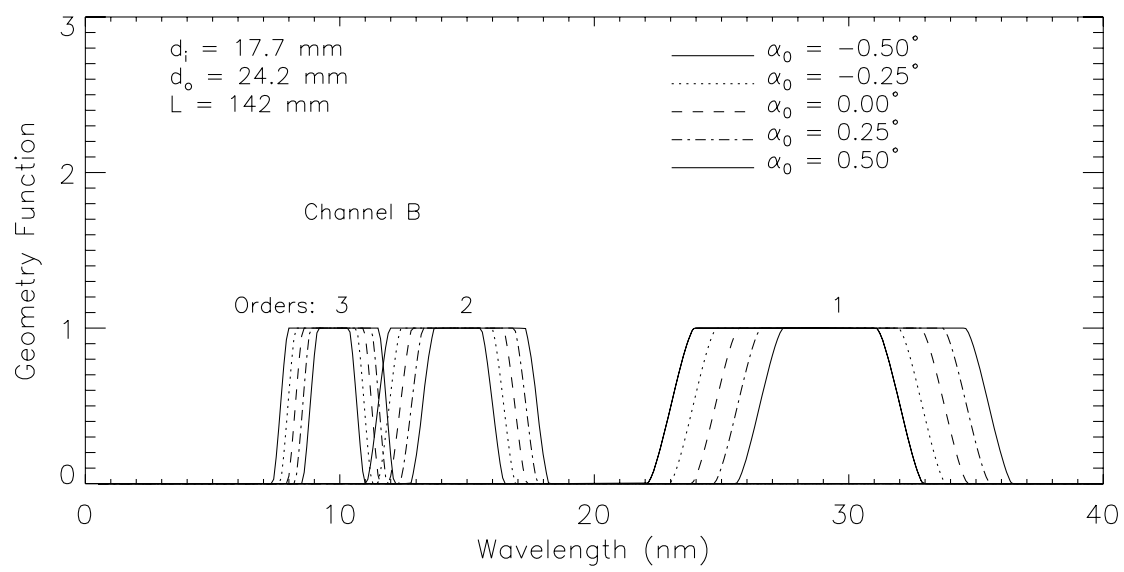

Figure 15 Geometry functions for Channel B out to 3rd order showing the effect of offsets from $\alpha_{\mathrm{O}}$ values of -0.50 to +0.50 .

at angles $\theta^{\prime}$ in the east-west direction and $\varphi^{\prime}$ in the vertical direction, then the geometry function can be represented as

$$
f_{\text {geom }}(\lambda)=\frac{\sum_{j=\theta_{j}^{\prime} \geq \theta_{i}^{\prime}}^{\theta_{j}^{\prime} \leq \theta^{\prime}} \sum_{k=1}^{M} g\left(\theta_{j}^{\prime}, \varphi_{k}^{\prime}\right)}{\sum_{j=1}^{N} \sum_{k=1}^{M} g\left(\theta_{j}^{\prime}, \varphi_{k}^{\prime}\right)}
$$

where $N$ is the total number of SXI pixels in the east - west direction, $M$ is the total number of pixels in the vertical direction, and $\theta_{i}^{\prime}$ and $\theta_{\mathrm{o}}^{\prime}$ are given by Equation (B.6).

\section{B.2.3. Illustration of Geometry Functions for Uniform Radiance}

Geometry functions for Channel B are shown in Figure 15. Orders $1-3$ are considered along with five $\alpha_{\mathrm{o}}$ values from $-0.5^{\circ}$ to $+0.5^{\circ}$. The selected range in $\alpha_{\mathrm{o}}$ is for illustration purposes and is not intended to reflect the true range under operational conditions that should become better characterized after PLT. Knowledge of the latter will await statistical studies of solar 
Table 10 Calculated conversion factors. Assumed spectral shapes within EUVS channels are taken from the SEE spectrum for TIMED orbit 24700 . The cross-disk normalized radiance distribution is assumed to be uniform. Included for each channel are changes in percent referenced to that channel's conversion factor with no offset. Original values to more significant figures were used to calculate the percentages.

\begin{tabular}{llllll}
\hline Channel & \multicolumn{5}{l}{ Conversion Factors in $10^{-9} \mathrm{~A} /\left[\mathrm{W} \mathrm{m}^{-2}\right]$} \\
\cline { 2 - 6 } & $\alpha_{\mathrm{O}}=-0.50^{\circ}$ & $\alpha_{\mathrm{O}}=-0.25^{\circ}$ & $\alpha_{\mathrm{O}}=0.00^{\circ}$ & $\alpha_{\mathrm{O}}=+0.25^{\circ}$ & $\alpha_{\mathrm{o}}=+0.50^{\circ}$ \\
\hline $\mathrm{A}$ & $0.681(-19 \%)$ & $0.760(-9.9 \%)$ & $0.843(0 \%)$ & $0.859(+1.8 \%)$ & $0.888(+5.3 \%)$ \\
$\mathrm{B}$ & $7.00(+0.5 \%)$ & $7.03(+1.0 \%)$ & $6.96(0 \%)$ & $7.30(+4.9 \%)$ & $8.23(+18 \%)$ \\
$\mathrm{C}$ & $5.17(-7.6 \%)$ & $5.37(-4.0 \%)$ & $5.59(0 \%)$ & $5.74(+2.7 \%)$ & $6.03(+7.8 \%)$ \\
$\mathrm{D}$ & $2.03(+8.9 \%)$ & $1.99(+6.4 \%)$ & $1.87(0 \%)$ & $1.73(-7.3 \%)$ & $1.58(-15 \%)$ \\
$\mathrm{E}$ & $2.64(-8.1 \%)$ & $2.88(+0.5 \%)$ & $2.87(0 \%)$ & $2.85(-0.7 \%)$ & $2.08(-28 \%)$ \\
\hline
\end{tabular}

offsets within SXI images. Values of the key parameters $d_{i}, d_{\mathrm{o}}$, and $L$ are listed in the figure (consistent with Table 9). Shifts in geometry functions like those shown in the figure can lead to important changes in conversion factors, to be quantified next.

\section{B.3. Changes in Conversion Factors Arising from Pointing Offsets}

Here, $\alpha_{\mathrm{o}}$ variations from $-0.5^{\circ}$ to $+0.5^{\circ}$ are considered using the non-flare SEE spectrum from TIMED orbit 24700 introduced in Figure 1. Table 10 shows conversion factors by channel for the five identified values of $\alpha_{0}$. In parentheses are percent differences referenced for $\alpha_{\mathrm{o}}=0^{\circ}$. To understand the variations requires examination of the product terms in Equation (2) from one $\alpha_{\mathrm{o}}$ value to the next.

It should be kept in mind that the contents of Table 10 will change if different spectral shapes are used. In turn, portions of the above discussion may need to be changed that are specific to details in the product distributions. In Appendix C, we hold $\alpha_{\mathrm{o}}$ fixed (at $0^{\circ}$ ) and address the effects caused by spectral changes.

\section{B.4. Changes in Conversion Factors Arising from Cross-Disk Radiance Variations}

We repeat the calculations just discussed but here for a non-uniform distribution taken from a GOES-13 SXI image. The image was recorded at 10:42 UT on 5 December 2006, near peak activity for the $\mathrm{X} 9$ flare on that day. There is a significant departure from uniformity in this image with the flare positioned on the eastern edge of the solar disk.

The effect of an asymmetric radiance distribution is manifested directly in the geometry function. Since the geometry function represents the fraction of the total disk intensity per $\mathrm{nm}$ at $\lambda$ incident on a transmission grating that is diffracted onto the detector, an asymmetric radiance distribution will have a noticeable effect at wavelengths where only part of the source radiance is diffracted onto the detector (i.e. at the wavelengths where the geometry function falls off from unity). If, for example, there is a bright region on the right limb of the solar disk, the geometry function for detectors with negative (positive) orders will experience a shift to shorter (longer) wavelengths as the geometry function drops from unity. Conversion factors for the non-uniform distribution under discussion appear in Table 11 for the same set of $\alpha_{\mathrm{o}}$ values as addressed in Table 10. Differencing the conversion factors in the two tables gives the percentages appearing within parentheses. In general, deviations from uniform emission across the solar disk will not require attention in the operational processing of EUVS data. 
Table 11 Similar to Table 10 except that uniform emission has been replaced by a highly asymmetric distribution taken from an SXI flare image (see text). The percentages are obtained by differencing Tables 10 and 11 (but using more significant figures than displayed) and then referencing the differences to the conversion factors in Table 10 .

\begin{tabular}{llllll}
\hline Channel & \multicolumn{2}{l}{ Conversion Factors in $10^{-9} \mathrm{~A} /\left[\mathrm{W} \mathrm{m}^{-2}\right]$} \\
\cline { 2 - 6 } & $\alpha_{\mathrm{O}}=-0.50^{\circ}$ & $\alpha_{\mathrm{O}}=-0.25^{\circ}$ & $\alpha_{\mathrm{O}}=0.00^{\circ}$ & $\alpha_{\mathrm{O}}=+0.25^{\circ}$ & $\alpha_{\mathrm{O}}=+0.50^{\circ}$ \\
\hline $\mathrm{A}$ & $0.748(+9.8 \%)$ & $0.833(+9.7 \%)$ & $0.843(0.0 \%)$ & $0.936(+8.9 \%)$ & $0.989(+11 \%)$ \\
$\mathrm{B}$ & $6.97(-0.4 \%)$ & $6.97(-0.9 \%)$ & $6.77(-2.8 \%)$ & $8.24(+13 \%)$ & $8.24(+0.1 \%)$ \\
$\mathrm{C}$ & $5.23(+1.2 \%)$ & $5.48(+2.1 \%)$ & $5.83(+4.1 \%)$ & $6.29(+10 \%)$ & $6.60(+9.4 \%)$ \\
$\mathrm{D}$ & $2.00(-1.6 \%)$ & $1.91(-4.1 \%)$ & $1.75(-6.5 \%)$ & $1.66(-4.3 \%)$ & $1.41(-11 \%)$ \\
$\mathrm{E}$ & $2.71(+2.7 \%)$ & $2.87(-0.4 \%)$ & $2.85(-0.5 \%)$ & $2.74(-3.8 \%)$ & $0.521(-75 \%)$ \\
\hline
\end{tabular}

Table 12 Conversion factors $C_{n}^{-1}$ and energy fluxes $Q_{n}\left(\Delta \lambda^{n}\right)$ for $\alpha_{\mathrm{o}}=0^{\circ}$ using the non-flare spectra in Figure 1. The $Q$ values are specified over the intervals given in the right-most column of Table 1 and for observations under non-flare conditions in early July 2006. Included for each channel are percent changes referenced to the two SEE columns (but using more significant figures than displayed).

\begin{tabular}{|c|c|c|c|c|c|c|c|}
\hline \multirow[b]{2}{*}{ Channel } & \multicolumn{3}{|c|}{$C_{n}^{-1}$ in $10^{8}\left[\mathrm{~W} \mathrm{~m}^{-2}\right] / \mathrm{A}$} & \multirow[t]{2}{*}{ Counts } & \multicolumn{3}{|c|}{$Q_{n}\left(\Delta \lambda^{n}\right)$ in $\mathrm{mW} \mathrm{m}^{-2}$} \\
\hline & $\begin{array}{l}\text { SEE } \\
\text { orbit } 24700\end{array}$ & NRLEUV & SOLERS22 & & $\begin{array}{l}\text { SEE } \\
\text { orbit } 24700\end{array}$ & NRLEUV & SOLERS22 \\
\hline A & 11.3 & $\begin{array}{c}11.2 \\
(-0.3 \%)\end{array}$ & $\begin{array}{r}6.99 \\
(-38 \%)\end{array}$ & 25547 & 0.204 & $\begin{array}{r}0.212 \\
(+3.9 \%)\end{array}$ & $\begin{array}{r}0.253 \\
(+24 \%)\end{array}$ \\
\hline B & 1.46 & $\begin{array}{c}1.60 \\
(+9.9 \%)\end{array}$ & $\begin{array}{r}1.18 \\
(-19 \%)\end{array}$ & 22227 & 0.742 & $\begin{array}{r}0.739 \\
(-0.4 \%)\end{array}$ & $\begin{array}{r}0.745 \\
(+0.5 \%)\end{array}$ \\
\hline $\mathrm{C}$ & 1.79 & $\begin{array}{c}1.69 \\
(-5.6 \%)\end{array}$ & $\begin{array}{c}1.71 \\
(-4.6 \%)\end{array}$ & 21979 & 0.223 & $\begin{array}{r}0.235 \\
(+5.5 \%)\end{array}$ & $\begin{array}{r}0.305 \\
(+37 \%)\end{array}$ \\
\hline $\mathrm{D}$ & 5.37 & $\begin{array}{c}5.04 \\
(-6.1 \%)\end{array}$ & $\begin{array}{c}5.86 \\
(+9.2 \%)\end{array}$ & 26755 & 2.26 & $\begin{array}{c}2.20 \\
(-2.6 \%)\end{array}$ & $\begin{array}{r}2.49 \\
(+10 \%)\end{array}$ \\
\hline
\end{tabular}

\section{Appendix C: Effects Arising from Variations in Spectral Shape}

It is much more difficult to assign uncertainties to conversion factors based on uncertainties in spectral shape than in $\alpha_{\mathrm{o}}$. Beyond the challenge of formulating a realistic range of spectra (ignoring overall magnitude shifts that are not relevant), there is limited knowledge of how the true spectral behavior over a given channel's wavelength domain changes with changing solar conditions, especially when a flare occurs. Here, our limited objectives are to first examine the range of conversion factor values obtained by using the spectral behavior taken from Figure 1, namely from SEE, NRLEUV, and SOLERS22 spectra under non-flare conditions. Secondly, SEE preflare and flare spectra are then considered from 28 October 2003 along with an NRLEUV flare spectrum in order to examine flare effects. $Q$ values are also addressed from measurements at a single point in time and, as noted earlier, refer to a spectral interval smaller than that required for determination of conversion factors. The exercise being described should provide insights into the magnitude of the problem where variability observed in broad-band solar energy fluxes may be, in part, caused by calibration changes arising from unknown spectral variability. 
Channel $\mathrm{E}$ is excluded from this discussion. Unlike the other channels, spectral variability plays essentially no role in specifying $Q$ values, since the observed region is thoroughly dominated by Ly $\alpha$ at $121.6 \mathrm{~nm}$.

\section{C.1. Non-flare Spectra}

We begin with Table 12, which shows the inverse of conversion factors, $C_{n}^{-1}$, for Channels A-D and for the three non-flare spectra in Figure 1. Also included are $Q_{n}\left(\Delta \lambda^{n}\right)$ values obtained from Equation (5) subsequent to the use of $C_{n}^{-1}$ in Equation (1) to first obtain $Q_{n}\left(\lambda_{\max }^{n}-\lambda_{\min }^{n}\right)$. Referring back to Section 1 , we recall that $\Delta \lambda^{n}$ is a subinterval of $\lambda_{\max }^{n}-\lambda_{\min }^{n}$ with both sets of intervals given in Table 1 . The needed counts displayed in the middle of the table are taken from the beginning of 1 July 2006. The date is not significant since the interest here is restricted to relative variations in $Q_{n}$ from one spectral shape to the next. For convenience in examining the variations in Table 12, changes in percent are shown under NRLEUV and SOLERS22 referenced to SEE. While percent variations in $Q_{n}\left(\lambda_{\max }^{n}-\lambda_{\min }^{n}\right)$ are the same as for $C_{n}^{-1}$ (see Equation (1)), this breaks down for $Q_{n}\left(\Delta \lambda^{n}\right)$ due to not sampling the full $\lambda_{\max }^{n}-\lambda_{\min }^{n}$ interval under changing spectral behavior. An example of this comes from the first row in Table 12 for Channel A where $C_{\mathrm{A}}^{-1}$ for SOLERS22 drops $38 \%$ while $Q_{\mathrm{A}}$ actually increases by $24 \%$. This is caused by a different change in scaled spectra from SEE to SOLER22 over $\Delta \lambda^{n}$ compared to the wider $\lambda_{\max }^{n}-\lambda_{\min }^{n}$ interval, which will become clearer in the following discussion.

We will return to Table 12 after introducing figures that provide insight into its content. The first of these is Figure 16, which contains four panels addressing Channel A. Three similar Figures 17, 18 and 19 address, in order, the remaining three channels of interest. In any of the four figures, the top panel displays normalized spectra for the three spectra of interest. For display purposes, the resolution is $2 \mathrm{~nm}$ (rather than $1 \mathrm{~nm}$, for which the calculations were performed), which applies to the remaining panels as well. Normalization is over the full signal range (see middle column in Table 1). The second panel displays the integrated response function for $\alpha_{\mathrm{o}}=0^{\circ}$. The third panel contains the products of the normalized spectra with the response function. For each product distribution, the sum gives the conversion factor for that distribution; in other words, the average response function weighted by the choice of spectral shape (always normalized). The three values are displayed in the upper right; their inverses appear in Table 12. The bottom panel shows absolute spectra obtained from Equations (1) and (4) using the number of counts displayed above the spectra (taken from Table 12). The shaded region identifies $\Delta \lambda^{n}$ from Table 1. Summing over this region gives the $Q_{n}$ values listed in the upper right, which also appear in Table 12. We have performed one extrapolation of the SOLERS22 spectrum. This can be seen in Figure 19 for Channel D, where the spectrum has been extended from 80 to $85 \mathrm{~nm}$ using the relative variation of the SEE spectrum over this region. The extrapolation has no impact on results for the given channel.

\section{C.1.1. Further Discussion of Table 12}

C.1.1.1. Channels $A$ and $B$ With the insight provided by the above spectral plots, we can now better understand the variations in Table 12. Starting with Channel A, the smaller $C_{n}^{-1}$ value for SOLERS22 (down 38\%) is seen to arise from larger values of its normalized spectrum (compared to SEE) over an extended region of relatively large response function values (see Figure 16). This leads to a drop in $Q_{\mathrm{A}}\left(\lambda_{\max }^{n}-\lambda_{\min }^{n}\right)$ by the same amount as obtained from Equation (1), which is also obtained by summing the entire green spectrum in the 

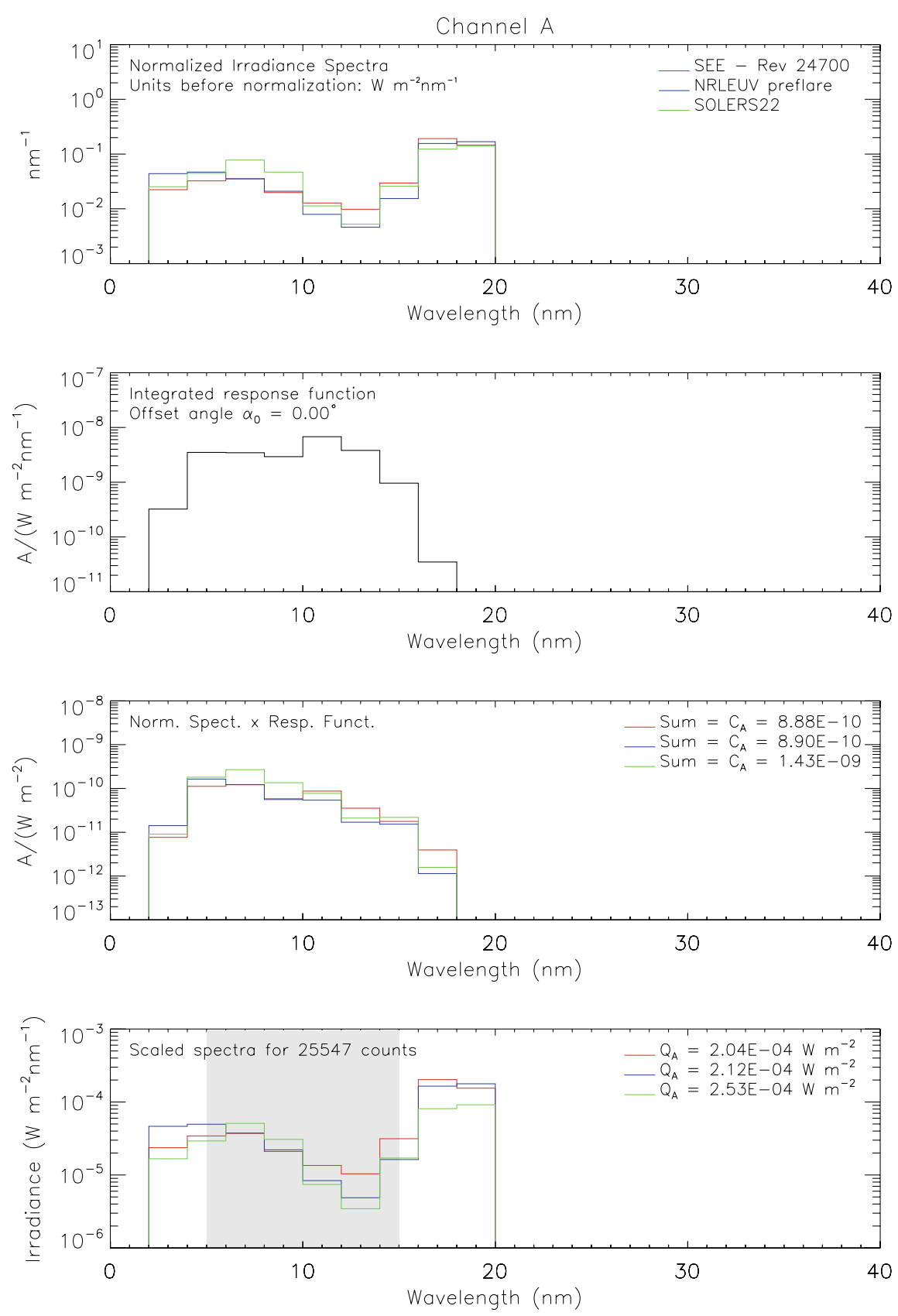

Figure 16 The contents illustrate how the conversion factor for Channel A responds to changes in spectral shape. Starting with the top panel are normalized spectra over the range relevant to this channel based on the non-flare spectra in Figure 1. The integrated response function appears in the second panel for $\alpha_{0}=0.0^{\circ}$. The products of this function with the normalized spectra are shown in the third panel along with their sums, namely, the corresponding conversion factors. In the bottom panel are scalings of the normalized spectra using the conversion factors with the counts displayed in the panel. A shaded region has been added that identifies the reporting interval from Table 1 along with $Q$ values over this interval. 

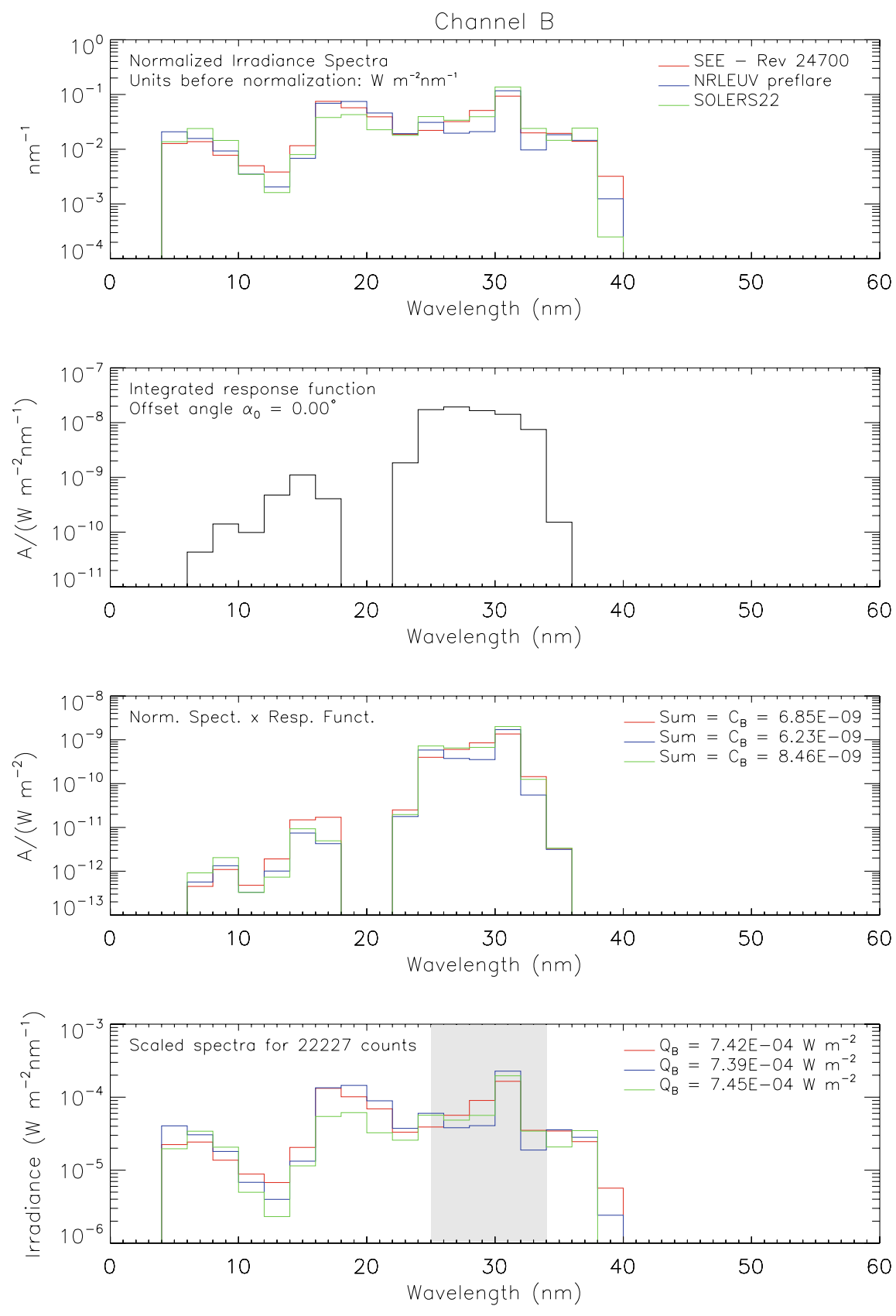

Figure 17 Similar to Figure 16 except for Channel B. 

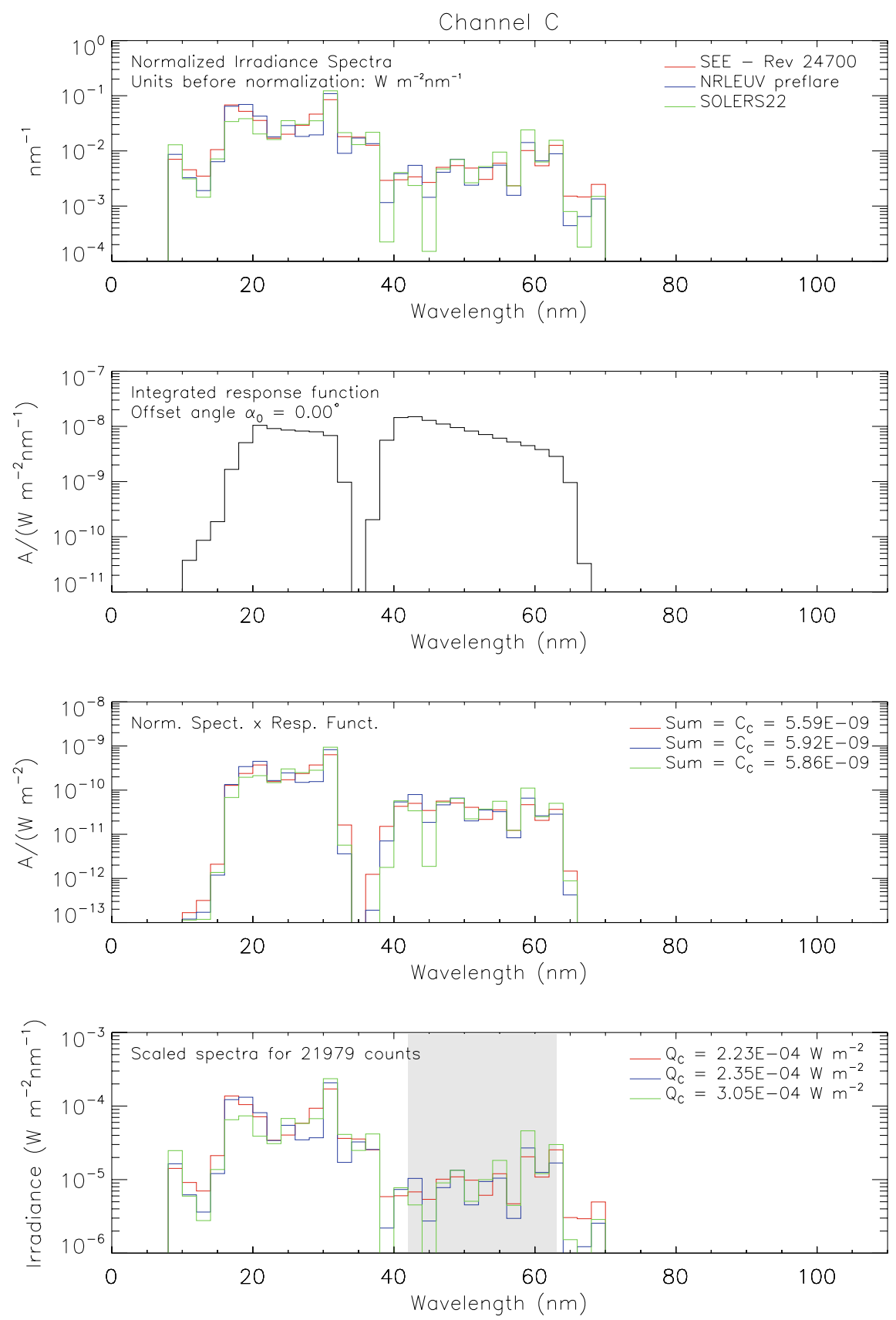

Figure 18 Similar to Figure 16 except for Channel C. 

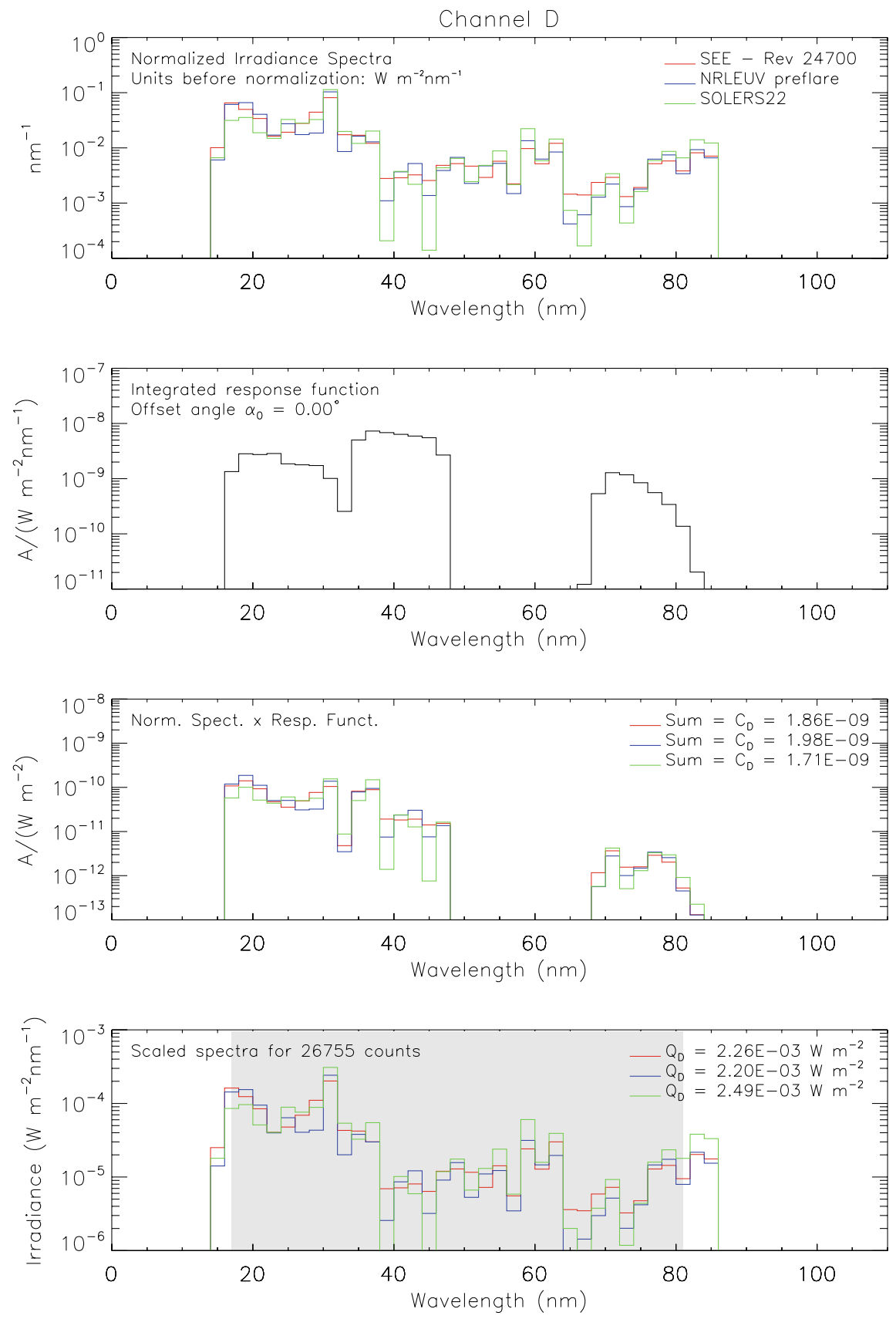

Figure 19 Similar to Figure 16 except for Channel D. 
bottom panel of Figure 16 (since the normalized spectrum is scaled by this $Q$ value). This decrease in the integrated spectrum, however, is not reflected in decreased spectral values at all wavelengths. Between 5 and $10 \mathrm{~nm}$, where SEE possesses its largest values within the shaded region, SOLERS22 exceeds these values and in turn leads to an increase in $Q$ over this region instead of the decrease over the full interval. Thus, we see that there is a complicated relationship between total signal and what is reported as an EUVS energy flux over a subinterval of the region producing this signal. Spectral plots like those in Figures 16-19 are essential in order to explore this relationship.

For Channel B, the normalized SOLERS22 spectrum compared to those for SEE and NRLEUV also produces larger integrand values in the region of maximum sensitivity that, in turn, leads to the $19 \%$ drop in $C_{n}^{-1}$ shown in the table. There is essentially no difference between the SOLERS22 $Q_{n}\left(\Delta \lambda^{n}\right)$ value and the other two, which again illustrates the lack of correlation with variations among the $C_{n}^{-1}$ values.

C.1.1.2. Channel $C$ This channel calls for special attention due to its reporting interval containing only a small fraction of the energy flux over the full interval. The $C_{n}^{-1}$ values for the three spectra are within about $6 \%$ of one another, which translates into the same agreement among the sums over the full spectra in the bottom panel of Figure 18 (i.e., $\left.Q_{\mathrm{C}}\left(\lambda_{\max }^{n}-\lambda_{\min }^{n}\right)\right)$. Within the shaded region, however, SOLERS22 $Q$ increases by $37 \%$ above its SEE counterpart. Such a large difference points to a weakness in selecting this region for reporting energy fluxes. It is sufficient to focus attention on the normalized spectra in Figure 18, since their relative differences are essentially preserved in the scaled spectra due to the similar $C_{n}^{-1}$ values. Firstly, the overall differences between SEE and SOLERS22 over $\Delta \lambda^{n}$ are subjected to a normalization process outside of this region, namely below $35 \mathrm{~nm}$ where most of the energy flux resides. Thus, normalization is not constraining the spectral sums over $\Delta \lambda^{n}$ to be similar, as it is below $35 \mathrm{~nm}$. Secondly, overall spectral shapes between SEE and SOLERS22 are different in going from the region shortward of $35 \mathrm{~nm}$ to the $\Delta \lambda^{n}$ region. There is greater relative energy flux for SOLERS22 compared to the region shortward of $35 \mathrm{~nm}$. Thus, it is not surprising that there can be a significant difference between $Q_{n}\left(\Delta \lambda^{n}\right)$ values, given these conditions. Generalizations cannot be made due to spectral complexity, its associated uncertainties, and the impact on $\phi_{\mathrm{EUVS}}^{n, j}$ (from Equation (4)) over $\Delta \lambda^{n}$ arising from normalizations and differences in $C_{n}^{-1}$ values. For example, the addressed change of $37 \%$ in $Q_{\mathrm{C}}\left(\Delta \lambda^{\mathrm{C}}\right)$ cannot be extrapolated to even a guess as to the uncertainty in Channel $\mathrm{C}$ energy fluxes arising from spectral uncertainties. If, for example, under non-flare conditions, we were using a spectral shape throughout the region of total signal that was physically realistic with only modest variations as activity changed, then the uncertainty would be modest compared to the change under discussion. There would then be no disadvantage to $\Delta \lambda^{n}$ spanning a region whose $Q_{n}$ value is associated with only a minor fraction of the signal. It does, however, place more demand for accurate spectral representation relative to where most of the signal resides.

C.1.1.3. Channel $D$ For this channel, there is much better agreement between changes in $C_{n}^{-1}$ and $Q_{n}\left(\Delta \lambda^{n}\right)$ and less spread in the latter quantity among the three spectra compared to Channel C. In Figure 19, the spectral region of total signal is seen to be very similar to that of Channel C. The big difference is the $\Delta \lambda^{n}$ region displayed in the bottom panel, which is wide enough to contain most of the signal. This assures $Q_{n}\left(\Delta \lambda^{n}\right)$ variations similar to those for $C_{n}^{-1}$. 

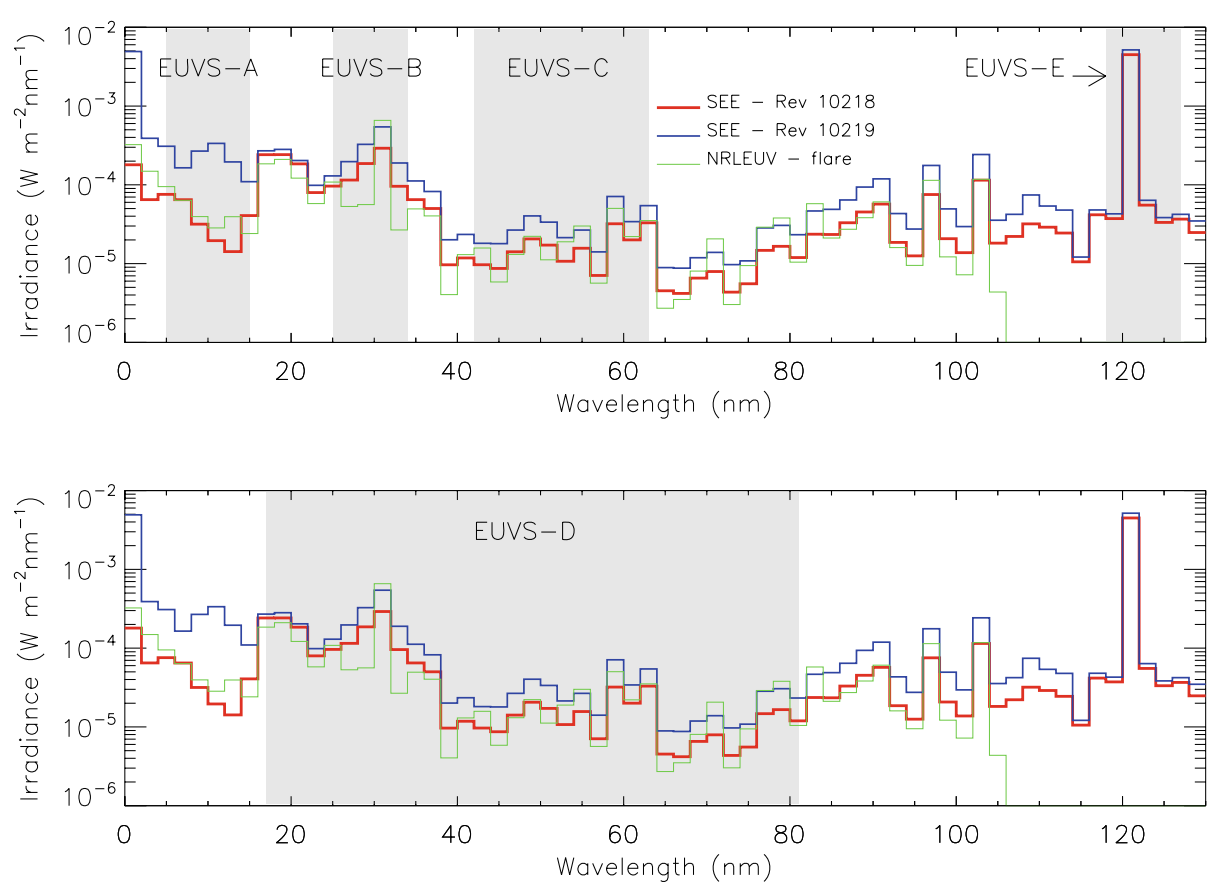

Figure 20 Similar to Figure 1 except for SEE preflare (orbit 10218), flare (orbit 10219), and NRLEUV flare spectra.

\section{C.2. Flare Spectra}

The discussion to follow will be briefer than in Section C.1, since much of that discussion applies here as well. In general, we are examining the effects caused by changes in spectral shape, whether under non-flare or flare conditions. As above, results are specific to a set of three spectra from which generalizations about uncertainties in energy fluxes should not be made, given such a limited sampling of spectral shapes and uncertainty about how physically realistic they are in their details, at least shortward of, say, $25 \mathrm{~nm}$. We begin with Figure 20, which shows SEE spectra from orbits 10218 and 10219 on 28 October 2003 along with an NRLEUV spectrum. SEE recorded observations near the peak of the X-class flare that occurred during orbit 10219 . Preflare conditions existed during orbit 10218 . The NRLEUV spectrum addresses flare conditions, but not conditions for the flare under discussion. The figure is similar to Figure 1 in that two panels with the same spectra are presented in order to clearly identify the intervals for reporting EUVS energy fluxes. The SEE spectra possess similar shapes longward of about $25 \mathrm{~nm}$. The most pronounced differences occur shortward of $16 \mathrm{~nm}$. The biggest differences in spectral shape between NRLEUV and SEE (orbit 10219 ) also occur shortward of about $16 \mathrm{~nm}$ where, in fact, NRLEUV behaves more similarly to the preflare SEE spectrum. One can already anticipate, based on the similarities in spectral shape longward of $16 \mathrm{~nm}$, that big differences will not be seen in $C_{n}^{-1}$ with the possible exception of Channel A.

Results similar to those in Table 12 are given in Table 13 for the spectra just introduced but without figures in the format of Figures 16-19. Such figures contain similar information and do not add significant insight beyond that already gained. $C_{n}^{-1}$ and $Q_{n}\left(\Delta \lambda^{n}\right)$ values 
Table 13 Similar to Table 12, except for two SEE spectra, one just before and one during the 28 October 2003 flare along with an NRLEUV flare spectrum. Use is made of the same counts as appear in Table 12 since interest here is restricted to relative variations from one spectral shape to the next.

\begin{tabular}{|c|c|c|c|c|c|c|}
\hline \multirow[t]{2}{*}{ Channel } & \multicolumn{3}{|c|}{$C_{n}^{-1}$ in $10^{8}\left[\mathrm{~W} \mathrm{~m}^{-2}\right] / \mathrm{A}$} & \multicolumn{3}{|l|}{$Q_{n}$ in $\mathrm{mW} \mathrm{m}^{-2}$} \\
\hline & $\begin{array}{l}\text { SEE-Preflare } \\
\text { orbit } 10218\end{array}$ & $\begin{array}{l}\text { SEE-Flare } \\
\text { orbit } 10219\end{array}$ & $\begin{array}{l}\text { NRLEUV } \\
\text { Flare }\end{array}$ & $\begin{array}{l}\text { SEE-Preflare } \\
\text { orbit } 10218\end{array}$ & $\begin{array}{l}\text { SEE-Flare } \\
\text { orbit } 10219\end{array}$ & $\begin{array}{l}\text { NRLEUV } \\
\text { Flare }\end{array}$ \\
\hline A & 9.71 & $\begin{array}{r}4.03 \\
(-59 \%)\end{array}$ & $\begin{array}{r}7.90 \\
(-19 \%)\end{array}$ & 0.196 & $\begin{array}{r}0.184 \\
(-6.1 \%)\end{array}$ & $\begin{array}{r}0.203 \\
(+3.2 \%)\end{array}$ \\
\hline B & 1.56 & $\begin{array}{r}1.81 \\
(+16 \%)\end{array}$ & $\begin{array}{r}1.33 \\
(-15 \%)\end{array}$ & 0.746 & $\begin{array}{r}0.765 \\
(+2.4 \%)\end{array}$ & $\begin{array}{r}0.757 \\
(+1.4 \%)\end{array}$ \\
\hline $\mathrm{C}$ & 1.76 & $\begin{array}{r}2.17 \\
(+23 \%)\end{array}$ & $\begin{array}{c}1.65 \\
(-6.1 \%)\end{array}$ & 0.174 & $\begin{array}{r}0.216 \\
(+25 \%)\end{array}$ & $\begin{array}{r}0.217 \\
(+25 \%)\end{array}$ \\
\hline D & 5.11 & $\begin{array}{c}5.48 \\
(+7.2 \%)\end{array}$ & $\begin{array}{c}5.58 \\
(+9.3 \%)\end{array}$ & 2.18 & $\begin{array}{c}2.29 \\
(+5.3 \%)\end{array}$ & $\begin{array}{r}2.42 \\
(+11 \%)\end{array}$ \\
\hline
\end{tabular}

for the orbit 10218 spectrum serve as references for the changes displayed in percent. The largest percent differences in $Q_{n}$ occur for Channel C, as expected from the above discussion under Section C.1.1.2.

\section{References}

Bailey, S.M., Woods, T.N., Barth, C.A., Solomon, S.C., Canfield, L.R., Korde, R.: 2000, J. Geophys. Res. 105, 27179.

Bailey, S.M., Woods, T.N., Barth, C.A., Solomon, S.C., Canfield, L.R., Korde, R.: 2001, J. Geophys. Res. 106, 15791.

Burns, A.G., Killeen, T.L., Roble, R.G.: 1989, J. Geophys. Res. 94, 3670.

Ceglio, N.M., Hawryluk, A.M., Stearns, D.G., Kühne, M., Müller, P.: 1988, Opt. Lett. 13, 267.

Chamberlin, P.C., Woods, T.N., Eparvier, F.G.: 2007, Space Weather 5(7), S07005. doi:10.1029/ 2007 SW000316.

Dickinson, R.E., Ridley, E.C., Roble, R.G.: 1984, J. Atmos. Sci. 41, 205.

Fuller-Rowell, T.J., Rees, D.: 1983, Planet. Space Sci. 31, 1209.

Guha, S., Jones, A., Benner, S.: 2007, In: Fineschi, S., Viereck, R.A. (eds.) Solar Physics and Space Weather Instrumentation II, Proc. of SPIE 6689, 66890L-1.

Henke, B.L., Gullikson, E.M., Davis, J.C.: 1993, At. Data Nucl. Data Tables 54, 181.

Hettrick, M.C., Cuneo, M.E., Porter, J., Ruggles, L.E., Simpson, W.W., Vargas, M.F., Wenger, D.: 2004, Appl. Opt. 43, 19.

Judge, D.L., McMullin, D.R., Ogawa, H.S., Hovestadt, D., Klecker, B., Hilchenbach, M., Moebius, E., Canfield, L.R., Vest, R.E., Watts, R., Tarrio, C., Khne, M., Wurz, P.: 1998, Solar Phys. 177, 161.

Judge, D.L., Ogawa, H.S., McMullin, D.R., Gangopadhyay, P., Pap, J.M.: 2000a, Adv. Space Res. 29(12), 1963.

Judge, D.L., Ogawa, H.S., McMullin, D.R., Gangopadhyay, P.: 2000b, Phys. Chem. Earth (C) 25, 417.

Lean, J.L., Warren, H.P., Mariska, J.T., Bishop, J.: 2003, J. Geophys. Res. 108, 1059. doi:10.1029/ 2001JA009238.

Mayr, H.G., Harris, I., Spencer, N.W.: 1978, Rev. Geophys. Space Phys. 16, 539.

McClintock, W.E., Rottman, G.J., Woods, T.N.: 2005, Solar Phys. 230, 225.

McClintock, W.E., Snow, M., Woods, T.N.: 2005, Solar Phys. 230, 259.

McMullin, D.R., Judge, D.L., Tarrio, C., Vest, R.E., Hanser, F.: 2004, Appl. Opt. 43, 3797.

Meier, R.R.: 1991, Space Sci. Rev. 58, 1.

Meier, R.R., Warren, H.P., Nicholas, A.C., Bishop, J., Huba, J.D., Drob, D.P., Lean, J.L., Picone, J.M., Mariska, J.T., Joyce, G., Judge, D.L., Thonnard, S.E., Dymond, K.F., Budzien, S.A.: 2002, Geophys. Res. Lett. 29, 1461.

Neupert, W.M.: 1968, Astrophys. J. Lett. 153, 59. 
Sofia, S., Heaps, W., Twigg, L.W.: 1994, Astrophys. J. 427, 1048.

Strickland, D.J., Lean, J.L., Daniell, R.E., Knight, H.K., Woo, W.K., Meier, R.R., Straus, P., Christensen, A.B., Woods, T.N., Aparvier, F.G., McMullin, D.R., Morrison, D., Paxton, L.J.: 2007, J. Geophys. Res. 112, A06313. doi:10.1029/2006JA012074.

Viereck, R., Hanser, F., Wise, J., Guha, S., Jones, A., McMullin, D., Plunkett, S., Strickland, D., Evans, S.: 2007, In: Fineschi, S., Viereck, R.A. (eds.) Solar Physics and Space Weather Instrumentation II, Proc. of SPIE 6689, 66890K-1.

Warren, H.P., Mariska, J.T., Lean, J.: 2001, J. Geophys. Res. 106, 15745.

Woods, T.N., Rottman, G.: 2005, Solar Phys. 230, 375.

Woods, T.N., Lean, J.L., Eparvier, F.G.: 2006, In: Gopalswamy, N., Bhattacharyya, A. (eds.) International Living With a Star (ILWS) Proceedings, Goa, India, 145. ISBN 81-87099-40-2.

Woods, T.N., Rottman, G., Vest, R.: 2005, Solar Phys. 230, 345.

Woods, T., Eparvier, F., Bailey, S., Solomon, S.C., Rottman, G., Lawrence, G., Roble, R., White, O.R., Lean, J., Tobiska, W.K.: 1998a, Proc. SPIE 3442, 180.

Woods, T., Ogawa, H.S., Tobiska, K., Farnil, F.: 1998b, Solar Phys. 177, 511.

Woods, T.N., Bailey, S.M., Peterson, W.K., Warren, H.P., Solomon, S.C., Eparvier, F.G., Garcia, H., Carlson, C.W., McFadden, J.P.: 2003, Space Weather 1, 1001.

Woods, T.N., Eparvier, F.G., Fontenla, J., Harder, J., Kopp, G., McClintock, W.E., Rottman, G., Smiley, B., Snow, M.: 2004, Geophys. Res. Lett. 31, L10802.

Woods, T.N., Eparvier, F.G., Bailey, S.M., Chamberlin, P.C., Lean, J., Rottman, G.J., Solomon, S.C., Tobiska, W.K., Woodraska, D.L.: 2005, J. Geophys. Res. 110, A01312.

Woods, T.N., Chamberlin, P.C., Peterson, W.K., Meier, R.R., Richards, P.G., Strickland, D.J., Lu, G., Qian, L., Solomon, S.C., Iijima, B.A., Mannucci, A.J., Tsurutani, B.T.: 2008, Solar Phys. 250, 235. doi:10.1007/s11207-008-9196-6. 